\title{
Overuse of Antibiotics and Antibiotic Resistance in Medical Applications Featuring Carbapenemase Resistant Enterobacteriaceae (CRE)
}

Nelson Kardos, PharmD, PhD*

International Technology Development Corporation, USA

Received: 15 September, 2017; Accepted: 30 October, 2017; Published: 01 November, 2017

*Corresponding author: Nelson Kardos, PharmD, PhD, International Technology Development Corporation, USA, Email: drnkardos@gmail.com

\begin{abstract}
The decreasing effectiveness of antibiotics in treating common infections results from the spread of antimicrobial resistance (AMR), and is building up to become an epic global public health crisis. Extended periods of antibiotic overuse and misuse since their introduction have applied strong selective pressure towards high-level AMR and Multiple Drug Resistance (MDR), rendering entire classes of antibiotics ineffective. The primary driving force for this global AMR pandemic is the widespread misuse and overuse of antibiotics, in both medical and nonmedical applications. Antibiotics have been misused in all of their applications, including [1]:
\end{abstract}

- Hospital and outpatient use by physicians through unnecessary, indiscriminate or incorrect prescribing.

- By patients, through incorrect dosing and course durations.

- $\quad$ Large-scale use in agriculture for disease treatment, prophylaxis and growth promotion in animal husbandry and food production.

These actions not only have provoked the emergence of resistant microbes, but also have provided optimal environments for the spread of and selection of resistance determinants. It has been established in many countries that the levels of antibiotic consumption consistently correlate with levels of antibiotic resistance (i.e. the more antibiotics are being used in a population, the more resistance to antibiotics there will be in bacteria responsible for infections in that population). Of particular concern is the development and spread of Carbapenem-resistant Enterobacteriaceae (i.e CRE) [2]. The global emergence of carbapenemase-producing organisms is a public health emergency because these enzymes confer resistance to both carbapenems and nearly all $\beta$-lactam antibiotics, and are often associated with multidrug or pandrug resistance $[3,4]$. Resistance to antibiotics mediated by acquired carbapenemase enzymes in gram-negative bacteria - principally the Enterobacteriaceae species is a serious public health concern. Most Carbapenemase- producing isolates of Enterobacteriaceae are resistant to multiple other classes of antibiotics, limiting therapeutic options to patients. Since the carbapenem antibiotics are the last line of defense against multidrug-resistant gram-negative bacterial infections, their vulnerability represents a real public health crisis. This article will focus on the overuse of antibiotics through medical applications.

\section{Antimicrobial Resistance (AMR): A Global Public Health Crisis}

The Age of Antibiotics: Prior to the discovery of penicillin as the first available antibiotic, infectious disease had been the leading cause of death throughout history. Penicillin was the first successful chemotherapeutic agent produced by microbes and it initiated the age of antibiotics. It represents the first therapeutic agent that destroyed bacteria in vivo, was not destroyed in the body, and was non-toxic to humans. Penicillin belongs to the beta-lactam class of antibiotics, which are the most successful natural product group used in chemotherapy. Later developed members of this antibiotic class which are widely used in medicine today include the orally active semisynthetic penicillins (ampicillin, amoxicillin) and the cephalosporins. It has been estimated that at the end of the nineteenth century, nearly one third of all deaths were due to infectious disease. By the end of the twentieth century, the death rate from all sources of infection dropped to levels well below $10 \%$ [3]. No other class of medicine has had a comparable cumulative impact on reducing death rates and increasing life spans [4].

The Spread of Antimicrobial Resistance (AMR): The world is now entering the post antibiotic age because of the growing problem of Antimicrobial Resistance (AMR). Infections from resistant bacteria have become more common and some pathogens have become resistant to multiple classes of antibiotics. The spread of AMR threatens to compromise the treatment of all infectious disease and is one of the most serious problems confronting both contemporary and future global public health. The World Health Organization (WHO) had proclaimed 
antimicrobial resistance the focus of World Health Day in 2011 in recognition of this serious and growing problem [8]. Based on studies of the costs of infections caused by antibiotic- resistant pathogens versus antibiotic susceptible pathogens, the annual cost to the US healthcare system of antibiotic resistant infections is $\$ 21$ to $\$ 36$ billion, and more than 8 million additional hospital days $[1,2]$.

The Global Struggle against AMR: The loss of effective antibiotic treatment will not only compromise the ability to control routine infectious disease, but will also prevent the treatment of infectious complications in patients with other disease states. The following advanced medical treatments are dependent on antibiotics to fight infections [5,6]:

- $\quad$ Cancer Chemotherapy: Cancer patients are at risk to develop serious infections when their white blood cell count is low. Such infections can be serious, and effective antibiotics are needed to protect cancer patients from complications and death.

- $\quad$ Surgery: Patients are at risk for infection from many surgeries including joint replacements, etc. Antibiotics are routinely given before surgery to prevent infection.

- Rheumatoid Arthritis (RA): This disease reduces the patient's immune system and increases the risk of infection. Since many medicines used to treat RA can weaken the immune system, effective antibiotics are needed to ensure that arthritis patients can continue receiving treatments.

- Dialysis for End Stage Renal Disease: Such patients have a weakened immune system and a higher risk for blood stream infections. Such infections are the leading cause of death in dialysis patients. Effective antibiotics ensure that dialysis patients will continue to receive life-long treatment.

- Organ and Bone Marrow Transplant: These patients receive complex surgery and have weakened immune systems. They are at high risk for infections and the use of effective antibiotics is essential. Without effective antibiotics, all of these procedures would have to be reduced. The reduction in antibiotic effectiveness from resistant pathogens leads to more difficult and costly treatments as well as greater morbidity and mortality and death. The Center for Disease Control (CDC) issued a landmark report in September 2013 advising that AMR has become one of the most serious healthcare threats both in America and globally [13]. The CDC reports that some 2 million people per year in the USA are contracting infections that are resistant to antibiotic treatment. And among such infections, there are more than 23,000 reported deaths per year as a result of AMR. It is estimated that Health-Care-Associated Infections (HAI) is reported at $5 \%$ in the USA and $7.1 \%$ in Europe. In the UK, infection rates range from $3.8 \%$ to $8.7 \%$. However, the infection rates in the developing world are much higher. The pooled presence of HAI in the developing world is estimated at $15.5 \%$ [7]. These estimates are regarded as both conservative and on the low end. Such prospects present a preview of a grim post antibiotic era. AMR has been seen to emerge soon after the deployment new antibiotics, reflecting their overuse in both medical and non medical applications.
AMR has generally been observed to emerge in the clinic within a decade or less after development of a new antibiotic [8].

The concern over AMR is not confined to the USA, but is recognized by major world governments and leading world health organizations. In the UK, Chief Medical Officer Dame Sally Davies had publicly spoken out in 2013 against family doctors over prescribing antibiotics, stating that they are contributing to a "public health catastrophe" $[8,9]$. The British Govt. published a five year antimicrobial resistance strategy in Sept 2013 with the objectives of slowing the development and spread of AMR [10]. This new program has three strategic aims: (i) Improve the knowledge and understanding of AMR; (ii) Conserve and steward the effectiveness of existing treatments; (iii) Stimulate the development of new antibiotics, diagnostics and novel therapies. In March 2016, the British Govt. published an updated report regarding drug-resistant infections [11].Where annual deaths attributed to AMR worldwide were compared to other major causes of death, including cancer, diabetes, etc. The report estimated that in 2015 there were 700,000 global deaths from AMR, compared to 8.2 million deaths from cancer. However, the report estimated that by 2050, AMR deaths would rise to 10 million per year, exceeding world cancer deaths. A summary of this data is presented in Table 1.

Table 1: Deaths Attributable to AMR Every Year Vs. Other Causes of Death

\begin{tabular}{|c|c|c|}
\hline Cause of Death & $\begin{array}{l}\text { Death Estimates } \\
\qquad \text { (2015) }\end{array}$ & $\begin{array}{l}\text { Death Estimates } \\
\text { (2050) }\end{array}$ \\
\hline AMR & $700,000(a)$ & $10,000,000$ \\
\hline Cancer & $8,200,000$ & -------- \\
\hline Cholera & 110,000 & ------ \\
\hline Diabetes & $1,500,000$ & ------- \\
\hline Diarrhoreal Disease & $1,400,000$ & -------- \\
\hline Measles & 130,000 & -------- \\
\hline $\begin{array}{l}\text { Road Traffic } \\
\text { Accidents }\end{array}$ & $1,200,000$ & -------- \\
\hline Tetanus & 60,000 & ------- \\
\hline \multicolumn{3}{|c|}{$\begin{array}{l}\text { (a) Low estimate Table assembled by author based on data } \\
\text { from [11] }\end{array}$} \\
\hline
\end{tabular}

\section{AMR Driving Forces}

AMR as Natural Evolution: AMR is the acquired ability of pathogens to withstand the actions of an antibiotic that kills all of its sensitive counterparts. This feature originally arises from random mutations in existing genes or from intact genes that serve a similar purpose. Exposure to antibiotics and other antimicrobial products (biocides) in humans and animals applies pressure that encourages resistance to emerge, and favoring naturally resistant strains and strains that have acquired resistance [12].

The specific meaning of the term 'AMR' depends on the 
context. The clinical definition used refers to the ability of a microbe (bacteria, virus, fungus or parasite) to survive concentrations of antibiotics that kill sensitive cells of the same strain. For every antibiotic there are sensitive microbial strains which are killed or inhibited by the drug, and there are naturally resistant strains. For a given antibiotic, there are some species of bacteria that are susceptible and others are not. Bacterial species that are not susceptible to a particular drug are 'naturally resistant.' But species that were once sensitive to an antibiotic but eventually became resistant to it have 'acquired resistance'. Acquired resistance affects a subset of the strains in the entire species, and varies with location. When a sensitive strain gains the ability to withstand the antibiotic, it is resistant to that antibiotic [12,13]. Some of the mechanisms that bacteria acquire to become resistant to antibiotics include; (i) Acquisition of genes coding for enzymes that destroy antibiotics (eg: betalactamases); (ii) Acquisition of efflux pumps that expel antibiotics from the bacterial cell; (iii) Mutations that produce altered cell walls with dysfunctional antibiotic binding sites; and (iv) Mutations that result in a decrease in the outer membrane channels antibiotics need to enter the bacterial cell $[12,13]$.

In biochemical terms, AMR means that a pathogen is less susceptible than its counterparts and may not respond to the antibiotic. The evolution of microbes is Darwinian; only the fittest survive change. Antibiotics represent an evolutionary challenge to microbes, which if not overcome will kill them. Resistance is not an on and off condition. Resistance exists as a gradient that reflects phenotypic and genotypic variations in large microbial populations. Different resistance mechanisms confer different levels of resistance. Low resistance levels are often overcome, but can also play an important role in the emergence of resistance. Currently used definitions of AMR do not take such diversity into account [12].

AMR arises by chance through mechanisms that may represent a history of natural competition among microbes. The mechanisms, genes, and pathways of antibiotic production and resistance help microbes compete for niches in nature. Therefore, AMR is a normal component of microbial life and represents a normal evolutionary phenomena. However, these natural evolutionary phenomena are amplified by the use, both appropriate and inappropriate, of antimicrobials (antibiotics, antifungals, antivirals and biocides).

Most microbes can be a source of resistant genes, but selection for AMR often takes place in non-pathogenic microbes, since they make up the majority of the microbial world. Resistant genes are often derived from existing essential genes. Resistant genes may also originate from antibiotic producing strains that are used to protect themselves from their own harmful products, or from natural protection mechanisms. Developing resistance to antibiotics increases each of the genes available to microbes to also import other genes. And this causes their evolutionary approximation. Once a microbe derives genetic tools from resistance, it can pass that gene onto its progeny by clonal replication; or to other microbes through horizontal gene transfer. Horizontal gene transfer - the movement of genetic material from one organism to another- is the primary mechanism by which bacteria acquire antibiotic resistance. Antibiotics promote this genetic exchange by inducing the transfer of conjugative elements [2,12-14]. Indeed, for any Gramnegative resistance issue, and especially for Enterobacteriaceae, one must also consider not just the spread of resistant strains but also the spread of their resistance genes between plasmids, and the spread of those plasmids between strains, species and genera. There is significant potential for transfer of bacteria and their resistance elements between reservoirs, as well as both to and from man, and from animal and environmental sources [8].

Antibiotic Misuse as a Primary Driver for AMR: The largest driver for development and spread of AMR is the overuse and misuse of antibiotics in both medicine and in agriculture. Overuse includes use of broad spectrum antibiotics in varied practice settings when the pathogens that cause the infection are not known. Such misdiagnosis is caused by a number of factors in healthcare settings, including: lack of knowledge by prescribers; prescriber attitudes; lack of effective diagnostics; and lack of current treatment guidelines. Overuse and misuse of antibiotics occur in both the hospital setting as well as in community / primary care and long-term care settings $[2,6,12,13]$. Studies indicate that nearly $50 \%$ of antibiotic use in hospitals is unnecessary or inappropriate [5]. This situation is not confined to the USA, as a study of antibiotic misuse in medium-sized Swiss hospitals showed that a high proportion of hospitalized patients received antibiotics (25\%), of which $47 \%$ were categorized as antibiotic misuse (defined in the study as an absence of an indication for such antibiotics) $[15,16]$. Misuse of broad spectrum antibiotics may be the largest single factor for the spread of AMR. Studies have shown that broad spectrum antibiotics make up $50 \%$ of all antibiotic prescriptions for adults and $40 \%$ of prescriptions for children $[17,18]$. Accordingly, it has been shown from data in Korea, Japan and Turkey that reduction in the use of broad spectrum antibiotics can be a step towards a credible solution to the control of AMR [16,19].

Inappropriate use also includes the use of sub inhibitory concentrations of antibiotics. Low concentrations of antibiotics can enrich for resistance genes in a population while having little effect on overall bacterial mortality. The tendency to mutate also increases upon exposure to sub-inhibitory concentrations of antibiotics. Low concentrations of antibiotics can also select for strains that increase expression of their existing resistance genes, further enhancing their resistance [12]. Although antibiotic resistance is mainly considered to be a clinical problem, antibiotic use and overuse is not restricted to clinical settings. The majority of antibiotics consumed in the world are used in farming and animal agriculture and related settings (aquaculture). Overuse of antibiotics in agriculture leads to the spread and crosstransmission of antimicrobial-resistant microbes between humans, between animals, and between humans and animals and the environment.

AMR is most often portrayed as an undesirable consequence of antibiotic abuse or misuse. But this explanation is not a complete picture. The rate of AMR emergence is related to all uses 
of these drugs, and not just to their misuse. The total quantity of antibiotics put out into the environment also plays a role in AMR. Selection for AMR is not confined to the human body, and is not limited to hospitals, clinics and farms. Selection takes place anywhere an antibiotic is present in natural environments, and especially in sewage and surface water sediments. Antibiotics are often found in the latter places coupled with high densities of large microbe populations. Large amounts of antibiotics and biocides end up in sewage sludge, which make them primary sources for development of AMR. Increasing amounts of antibiotics and biocides that are found in waste water, sediment and sludge discharges originate from agricultural applications. The stability of an antibiotic is one key as to how it will impact development of AMR in the environment. Stable antibiotics are more likely to persist long enough to select for resistance $[12,13]$.

\section{Summary of AMR Drivers}

Travel and Trade: The growth of international travel and global trade serves to accelerate the movement of antibiotic resistance genes and strains around the world. Antibiotic resistant pathogens move around the world as quickly as people do. Travelers can contract and bring home resistant strains, both as active infections or as unaffected carriers. This aspect of AMR is discussed further in CRE - Solution: Prudent Use of Antibiotics in Healthcare.
Antibiotics as environmental contaminants: Selection for AMR can take place anywhere antibiotics are present. Large amounts of antibiotics are found in urban waste water as well as sediment and sewage sludge originating from agricultural applications. They can have significant effects on an ecosystem's stability and the selection for AMR. This aspect of AMR is discussed further in Section: Conclusion.

Overuse of antibiotics for medical applications: In healthcare settings, the overuse and inappropriate use of antibiotic are the primary drivers for development of AMR. Factors that contribute to poorly managed use of antibiotics in all healthcare settings include: (i) Poor surveillance of resistant pathogens; (ii) Insufficient antibiotic susceptibility testing and use of diagnostics, and (iii) Insufficient control of nosocomial infections and poor infection control, and (iv) Inappropriate prescribing of antibiotics for out-patient use [6,12].

Overuse of antibiotics in agricultural and veterinary applications: Antibiotics are widely used in agricultural settings, including in animals for both infection control and as growth promoters, and also in veterinary hospitals. All of these applications provide an opportunity for gene transfer and development of AMR $[12,13]$ and are discussed further in CRE Solution: Antibiotic Stewardship Programs.

Figure 1 shows the effect of direct selective pressure as a primary driving force for AMR. The figure illustrates that $\beta$-lactamases evolve with the appearance and use of each new antimicrobial class.

\title{
Beta-lactamase Evolve with Each New Antimicrobial Class
}

\author{
Wild Type Pathogen \\ Overuse of Penicillins \\ (Results in Beta-Lactamases, increasing the need for BLIC (Beta-lactam inhibitors) and Cephalosporins) \\ Beta-Lactamase \\ Overuse of Cephalosporin \& BLICs (Beta-Lactam Inhibitors) \\ $\prod$ (Results in ESBLs (Extended Spectrum Beta-Lactamase), increasing the need for Carbapenems) \\ ESBLs (EX: TEM. SHV. CTX-M, AmpC) \\ Overuse of Carbapenem \\ (Results in Carbapenemases - capable of hydrolyzing all Beta-lactams and Beta-lactamase inhibitors) \\ Carbapenemases (Ex: KPC, MBL, NDM-1)
}

\begin{abstract}
The evolution of Beta-lactamases with application of successive classes of antibiotics shows the development of AMR as a function of overuse of the same antibiotic. Overuse gives resistant clones an increased chance of establishing themselves in the host.
\end{abstract}

Figure 1: Selective Pressure as a Primary Driving Force for Antimicrobial Resistance (AMR) 


\section{Center for Disease Control (CDC) Threat Assessment for Healthcare \\ Acquired Infections (HAI)}

The decreasing effectiveness of antibiotics in treating common infections results from the spread of Antimicrobial Resistance (AMR) and is building up to become an epic global public health crisis. The crisis of reduced effectiveness of antibiotics to treat infections because of increasing AMR have been dramatically publicized in recent years by international health organizations (WHO) as well as Central Govt. Agencies (NHS in UK, CDC in USA) $[6,10,1]$. Extended periods of antibiotic overuse and misuse since their introduction have applied strong selective pressure towards high level AMR and Multiple Drug Resistance (MDR), rendering entire classes of antibiotics ineffective. The traditional response to AMR had been the introduction of new classes of antibiotics, a strategy which did not solve the problem but only bought a brief reprieve. However, over the last twenty years, there has been a significant decline in development and clinical introduction of new antibiotics to keep pace with the escalation of global AMR [14]. The rapid evolution and spread of AMR is illustrated in the case of the Beta-Lactam class of antibiotics. There are nearly 1,000 resistant related Beta lactamases that inactivate these antibiotics that have been identified, and have spread worldwide. The primary driving force for this global AMR pandemic is the widespread misuse and overuse of antibiotics, in both medical and non-medical applications. The introduction of every antibiotic product has been closely followed by emerging resistance to that antibiotic [6]. Levels of antibiotic consumption correlate with levels of AMR. Antibiotics have been misused in all of their applications, including:

- Hospital and outpatient use by physicians through unnecessary, indiscriminate or incorrect prescribing

- Out patients, through incorrect dosing and therapy course durations

- Large scale use in agriculture for disease treatment, prophylaxis and growth promotion in animal husbandry and food production

These actions not only have provoked the emergence of resistant microbes, but also have provided optimal environments for the spread of and selection of resistance determinants. It has been established in many countries that the levels of antibiotic consumption consistently correlate with levels of antibiotic resistance (i.e. the more antibiotics are being used in a population, the more resistance to antibiotics there will be in bacteria responsible for infections in this population). The increase in resistance from overuse of antibiotics in turn leads to cross transmission of AMR microbes between humans, between animals and between humans and animals and the environment. The two major areas for managing control and prevention of AMR are: (i) Prudent use of antibiotics: Use antibiotics only when needed, and with the correct dose, at the correct dose intervals,

Table 2: CDC Levels of Concern - Prioritization of Resistant Bacteria

\begin{tabular}{|c|c|c|}
\hline Urgent Threats & Serious Threats & Concerning Threats \\
\hline \multirow{2}{*}{ Clostridium difficile } & \multirow{2}{*}{ Drug-resistant Acinetobacter } & Vancomycin-resistant \\
\hline & & Staphylococcus aureus (VRSA) \\
\hline $\begin{array}{l}\text { Carbenapenem-resistant, } \\
\text { Enterobacteriaceae (CRE) }\end{array}$ & Drug-resistant Campylobacter & Erythromycin-resistant, Streptococcus Group A \\
\hline \multirow{11}{*}{ Drug-resistant Neisseria gonorrhoeae } & \multirow[b]{2}{*}{ Fluconazole-resistant Candida } & Clindamycin-resistant \\
\hline & & Streptococcus Group B \\
\hline & $\begin{array}{l}\text { Extended-spectrum, cephalosporin-resistant } \\
\text { Enterobacteriacease }\end{array}$ & \\
\hline & Vancomycin-Resistant Enterococcus (VRE) & \\
\hline & Drug-resistant Pseudomonas aeruginosa & \\
\hline & Drug-resistant nontyphoidal Salmonella & \\
\hline & Drug-resistant Salmonella typhi & \\
\hline & Drug-resistant Shigella & \\
\hline & $\begin{array}{l}\text { Methicillin-Resistant Staphylococcus Aureus } \\
\text { (MRSA) }\end{array}$ & \\
\hline & Drug-resistant Streptococcus pneumoniae & \\
\hline & Drug-resistant tuberculosis (MDR \& XDR) & \\
\hline \multicolumn{2}{|l|}{ Notes: MDR = Multiple Drug Resistance } & \\
\hline
\end{tabular}


and for the correct duration; and (ii) Hygienic precautions for control of infections [12].

In September 2013, the Center of Disease Control (CDC) in the USA published a lengthy study describing the scope of AMR in the USA, and recommending an action plan to contain the problem ("Antibiotic Resistant Threats 2013"- Tom Frieden, CDC Director)[1]. CDC declared AMR to be a very serious public health issue. The CDC estimates that more than 2 million people per year contract AMR infections and resulting in at least 23,000 direct deaths, and another 100,000 deaths from related complications. Similarly the European Center for Disease Prevention and Control reports that 25,000 people die each year in Europe from antibiotic resistant bacteria. This problem is also present in other parts of the world. For example, in India over 58,000 people die in one year from antibiotic resistant infections. In Thailand, antibiotic resistance causes more than 38,000 deaths per year and 3.2 million hospitalization days [18].These are minimum estimates based on available data and believed to represent an under reporting of cases.

The cost of AMR infections goes beyond patient deaths. The consequences include greater morbidity and healthcare expense. The impact of AMR on the American healthcare system is enormous. Almost two million Americans per year develop Hospital Acquired Infections (HAI), resulting in 99,000 deaths, most of which are to antibiotic resistant pathogens. It has been estimated that the annual cost to the American healthcare system of antibiotic- resistant pathogens versus antibiotic-susceptible pathogens is $\$ 21$ to $\$ 34$ billion/year and more than 8 million additional hospital days $[15,20]$. The vast majority of these HAI related deaths are due to AMR infections. HAI incidence is reported at approximately 5\% in the USA and 7.1\% in Europe, and pooled infection rates in the developing world are estimated at $15.5 \%[13,14]$. Based on studies of the costs of infections caused by antibiotic resistant pathogens vs. antibiotic susceptible pathogens, the annual cost to the US health system of antibiotic resistant infections is in the range of $\$ 21$ to $\$ 34$ billion, and 8 million additional hospital days [15]. As AMR increases, HAI becomes an increasing threat to patient welfare. The CDC has listed the hazard level of threat for AMR into three categories: Hazard Level Urgent; Hazard Level Serious; and threat Level Concerning. The pathogens covered in these threat levels are summarized in Table 2.

\section{ESKAPE Pathogens \& ESBL}

A select group of pathogens are responsible for the majority of HAI; acronymically termed the ESKAPE pathogens by IDSA (Infectious Disease Society of America $[15,23]$. The term ESKAPE indicates that these pathogens are capable of escaping the biocidal actions of antibiotics and collectively represent new paradigms in pathogenesis, transmission, resistance and severity of their infections. The ESKAPE acronym consists of [16]:

Enterococcus faecium

$S$ Staphylococcus aureus

K Klebsiella pneumoniae

\section{A Acinetobacter baumannii}

\section{P Pseudomonas aeruginosa}

E Enterobacter Species and includes ESBL (ExtendedSpectrum beta lactamase)

ESKAPE indicates that these bacteria have developed defenses that permit them to escape the biocidal actions of available and effective antibiotic therapies. All of the ESKAPE pathogens produce beta lactamase enzymes and have developed resistance against carbapenems. A brief highlight of the more important carbapenemase producing pathogens follows:

- $\quad$ Klebsiella pneumoniae: This pathogen is a member of the Gram-negative Enterobacteriacae family, and is prevalent worldwide in both hospital and community infections. This pathogen is recognized for accumulation and rapid dissemination of MDR determinants. And over the past decade, it has acquired an extensive range of $\beta$-lactamase enzymes capable of hydrolyzing the $\beta$-lactam ring common in penicillins, cephalosporins and also carbapenems. The development and spread of carbapenemresistant K. pneumoniae (CRKP) threatens the clinical effectiveness of $\beta$-lactams, fluoroquinolones and aminoglycosides [19].

- Acinetobacter baumannii: This is a Gram-negative opportunistic pathogen most often encountered in intensive care units and surgical wards, where extensive antibiotic use has enabled selection for AMR. This pathogen can grow across a range of temperatures, PHs and nutrient levels which make it highly adapted to survival in both human and environmental vectors and carries high rates of nosocomial cross-contamination. This pathogen is intrinsically resistant to antibiotics. It has acquired a broad range of $\beta$-lactamases, including carbapenemases. The broad acquisition of ESBLs gives some isolates resistance to all antibiotics, including imipenem and colistin [20].

- Pseudomonas aeruginosa: This pathogen is a Gramnegative facultative anaerobe. It preferentially colonizes immunocompromised patients and is known as an opportunistic pathogen associated with cancer patients and burn victims. It is resistant to fluoroquinolones through point mutations. In addition, this pathogen harbors broad-spectrum ESBLs including carbapenemases, which make it difficult to employ suitable empirical antibiotic therapies [20].

- $\quad$ Enterobacter Species and includes ESBL (ExtendedSpectrum beta lactamase). These species most commonly infect the urinary and respiratory tracts, but also cause bloodstream infections. They are shown to display broad MDR via plasmidencoded ESBLs and carbapenemases. Beside colistin and tigecycline, few antibiotics are effective against these resistant pathogens [23].

The ESKAPE organisms are responsible for a substantial percentage of nosocomial infections in hospitals and represent the vast majority of isolates whose resistance to antibiotics present serous treatment limitations. Within the ESKAPE pathogens, the problem of carbapenem resistant Enterobacteriaceae (CRE) 
infections are becoming the leading healthcare infection problem, and are referred to as "nightmare bacteria" by CDC director Dr. Tom Frieden [1]. The CRE pathogens include carbapenem resistant Klebsiella species and carbapenem resistant E. coli, resulting in 9,000 drug resistant infections /year and 600 deaths. CRE infections have become resistant to nearly all antibiotics in use today, including carbapenems - the antibiotic of last resort. CRE infections most commonly occur among patients who are receiving treatment for other conditions and whose care requires devices like ventilators, urinary catheters or intravenous catheters, and also patients taking long courses of antibiotics. Up to one half of patients die from CRE bloodstream infections $[21,22]$.

Extended spectrum $\beta$-lactamases (ESBLs) are enzymes produced by a variety of gram negative bacteria which confer an increased resistance to commonly used antibiotics $[22,23] . \quad \beta$-lactamases are hydrolytic enzymes which cleave the $\beta$-lactam ring and the primary mechanism of conferring bacterial resistance to $\beta$-lactam antibiotics such as penicillins and cephalosporins. These enzymes can be carried on bacterial chromosomes or may be plasmid mediated with the potential to move between bacterial populations. This ability of ESBL genes to jump between organisms leads to outbreaks of infections where easily transmissible pathogens are involved. Moreover, organisms that produce ESBLs have the capacity to acquire resistance to other antimicrobial classes such as quinolones, tetracyclines, cotrimazole, trimethoprim and aminoglycosides, which further limits therapeutic options. ESBLs have become a major cause of hospital-acquired infections and particularly in the Intensive Care Unit (ICU), where the majority of ESBL infections are isolated from critical care patients [23].

\section{CRE - Definition and Classification}

Introduction: Carbapenemases represent the most versatile family of $\beta$-lactamases, with the widest spectrum of activity among all the $\beta$-lactam-hydrolyzing enzymes. Although known as "carbapenemases" many of these enzymes recognize almost all hydrolysable $\beta$-lactams, and most are resistant against inhibition by all commercially viable $\beta$-lactamase inhibitors $[24,25]$. Carbapenemases are produced by Enterobacteriaceae bacteria (Carbapenem resistant Enterobacteriaceae = CRE), which include more than 70 different genera covering many different mechanisms causing carbapenem resistance. CRE are included in a broader category of CP-CRE- standing for carbapenemaseproducing CRE. CP-CRE is a subset of all CRE. All CRE are likely multidrug-resistant organisms for which interventions are required in healthcare settings to prevent transmission [24]. Carbapenemase-producing pathogens cause infections that are difficult to treat and have high mortality rates, due to their appearance in multidrug resistant pathogens such as Klebsiella.

pneumoniae, P. aeruginosa, and Acinetobacter spp, It has been found that carbapenemase genes are easily transferred on mobile elements among bacterial species, leading to the spread of infections. It should be noted that CRE that do not produce carbapenemases are generally still resistant to multiple antibiotics and are a serious health problem.
CDC Definition of Multiple Drug resistant Organisms (MDR0): The expert consensus of MDRO as microbial species resistant to multiple antimicrobial agents contain the following subsets and apply to CP and CRE infections:

- $\quad$ PDR: (Pan Drug resistance) non susceptible to all agents in all antimicrobial drug categories

- $\quad$ MDR: (Multi Drug Resistance)an isolate non susceptible to a minimum of one agent in at least three drug categories

- XDR: (Extensive Drug Resistance) an isolate is non susceptible to at least one agent in all but two or less antimicrobial drug categories [24,25].

CDC Definition of Carbapenemases: Previously, the CDC defined CRE as "Being non-susceptible to the carbapenems 'imipenem,' 'meropenem' or 'doripenem,' and resistant to all third generation cephalosporins tested." The CDC has adopted a new definition since January 2015 for CRE to read: "Being non-susceptible to the carbapenems 'imipenem,' 'meropenem' $O R$ 'doripenem,' or ertapenem, or documentation that the isolate possesses a carbapenemase [24]." The previous CDC CRE definition was designed to be more specific for CP-CRE. However, that definition was found to be complicated, difficult to implement and also missed some CP-CRE. Other reasons for the change in the CDC definition were (i) ertapenem was included to increase the ability to detect carbapenemase-producing strains compared to the previous CDC CRE definition; (ii) third generation cephalosporins are not included from the current CDC definition to simplify the definition, facilitate application and also accommodate the emergence of OXA-48-type producing CRE which might not be resistant to this class of antimicrobials [24].

Classification of $\boldsymbol{\beta}$-lactamases: More than 1,000 $\beta$-lactamases exist in gram-negative bacteria. Production of $\beta$-lactamases is the most widespread cause of carbapenem resistance. Two classification schemes exist for $\beta$-lactamases. One scheme is based on molecular classification and puts all $\beta$-lactamases into four distinct classes (A through D) based on amino acid sequence homology- the Ambler classes, also called 'Molecular Classes.' The other scheme is based on functional classification using substrate and inhibitor activity (classes 1 through 4) - the Bush-Jacoby classes, also called 'Functional Classes' [26-28]. This article will use the Ambler classification scheme and is depicted in Table 3.

Molecular classes A, C and D contain serine in their active site, while Group B contains zinc in their active site. Carbapenemases are found in Classes A, B \& D.

Class A $\boldsymbol{\beta}$-lactamase: They have serine active sites. The more important enzyme groups are: NMC, IMI, SME, KPC \& GES. $\mathrm{KPC}$ is the most common carbapenemase in the USA. While first isolated add from $K$. pneumoniae, it has since spread to other Enterobacteriacea.

Class B Beta-lactamase: They are also referred to as MBL (metallo- $\beta$-lactamases because they require the presence of zinc to function. The more important enzymes are: IMP, VIM, GIM and 
Table 3: Groupings of Carbapenemases and AmpC Beta-lactamases Within Beta-lactamase Classifications

\begin{tabular}{|c|c|c|c|c|}
\hline Ambler Group & $\begin{array}{l}\text { Bush Jacob } \\
\text { Group }\end{array}$ & Common Name & Mediates Resistance to & Representative Enzymes \\
\hline A & $2 \mathrm{f}$ & Serine carbapenemase & $\begin{array}{c}\text { Carbapenems; Penicillins; Cephalosporins; } \\
\text { Aztreonam }\end{array}$ & KPC, GES, SME1 \\
\hline B & $3 a$ & $\begin{array}{l}\text { Metallo- } \beta \text {-lactamases } \\
\text { (MBLs) }\end{array}$ & All $\beta$-lactams except aztreonam & IMP, NDM, VIM \\
\hline $\mathrm{C}$ & 1 & $\begin{array}{l}\text { Serine } \\
\text { cephalosporinase }\end{array}$ & Penicillins; Cephalosporins & AmpC \\
\hline $\mathrm{D}$ & $2 \mathrm{df}$ & Carbapenemase & $\begin{array}{c}\text { Carbapenems; Penicillins; Cephalosporins; } \\
\text { Aztreonam }\end{array}$ & OXA \\
\hline
\end{tabular}

Notes: $[27-29,48]$

$\mathrm{KPC}=$ Klebsiella pneumoniae carbapenemase

GES = Guiana extended spectrum

AmpC $=$ Cephalosporinase

SMEI = Serratia marcescens enzyme

IMP = Imipenem-hydrolyzing- $\beta$-lactamase

$\mathrm{VIM}=$ Verona integron-encoded metallo- $\beta$-lactamase

NDM $=$ New Delhi metallo- $\beta$-lactamase

OXA = Oxacillinase-hydrolyzing- $\beta$-lactamase

Table 4: Commonly Detected Carbapenemase in Enterobacteriaceae

(Groupings of Carbapenemases and AmpC Beta-lactamases Within Beta-lactamase Classifications)

\begin{tabular}{|c|c|c|c|c|}
\hline $\begin{array}{c}\text { Ambler } \\
\text { Group }\end{array}$ & $\begin{array}{c}\text { Bush- } \\
\text { Jacoby } \\
\text { Group }\end{array}$ & Common Name & Mediates Resistance to & $\begin{array}{c}\text { Representative } \\
\text { Enzymes }\end{array}$ \\
\hline A & $2 \mathrm{f}$ & Serine carbapenemase & Carbapenems; Penicillins; Cephalosporins; Aztreonam & KPC, GES, SME1 \\
\hline B & $3 \mathrm{a}$ & Metallo- $\beta$-lactamases (MBLs) & All $\beta$-lactams except aztreonam & IMP, NDM, VIM \\
\hline C & 1 & Serine cephalosporinase & Penicillins; Cephalosporins & AmpC \\
\hline D & $2 \mathrm{df}$ & Carbapenemase & Carbapenems; Penicillins; Cephalosporins; Aztreonam & OXA \\
\hline
\end{tabular}

Notes: $[27-29,48]$

$\mathrm{KPC}=$ Klebsiella pneumoniae carbapenemase

$\mathrm{GES}=$ Guiana extended spectrum

AmpC $=$ Cephalosporinase

SMEI $=$ Serratia marcescens enzyme

IMP = Imipenem-hydrolyzing- $\beta$-lactamase

VIM $=$ Verona integron-encoded metallo- $\beta$-lactamase

NDM $=$ New Delhi metallo- $\beta$-lactamase

OXA $=0$ xacillinase-hydrolyzing- $\beta$-lactamase

SPM. Until 2009, VIM subtype was the most widespread MBL. It has since been displaced in ranking by NDM-1 which is now the most globally prevalent MBL subtype.
Class C $\boldsymbol{\beta}$-lactamase: They have a serine active site. The important enzymes are AmpC. AmpC genes on the bacterial chromosomes produce low levels of $\beta$ - lactamases (repressed). When "de-repressed" the BL is hyper produced. AmpC BL has minimal activity against carbapenems and monobactams.

Citation: Kardos N (2017) Overuse of Antibiotics and Antibiotic Resistance in Medical Applications Featuring Carbapenemase Page 8 of 21 Resistant Enterobacteriaceae (CRE). SOJ Microbiol Infect Dis 5(5):1-21. 
However, when AmpC is combined with other mechanisms for reduced cell susceptibility, clinically significant levels of resistance are achieved Class $C \beta$ - lactamase are not generally classified as carbapenemases [28].

Class D $\boldsymbol{\beta}$-lactamase: This class has a serine active site. The important $\beta$-lactamase are of the oxacillanase (OXA) enzyme type. They have weak activity against carbapenems and are found primarily in $P$ aeruginosa and Acinetobacter. The major concern with OXA carbapenemases is their ability to rapidly mutate and expand their spectrum of activity [32].

Table 4 shows substrate and inhibition profiles of the more important carbapenemases -based on both the Ambler and BushJacoby classification schemes

Clinically Important Carbapenemases: The most common carbapenemases in Enterobacteriacea are displayed in Table 4. They are: KPC;VIM; NDM and OXA-48 [31,32].

KPC: Klebsiella pneumoniae carbapenemase is a class " $\mathrm{A}$ " $\beta$-lactamase. It can hydrolyze penicillins, cephalosporins and carbapenems. KPC was first reported in North Carolina in 1996, and has since spread globally. The KPC gene can be acquired by other species of Enterobacteriacea including Enterobacter spp. and Escherichia coli and on rare occasions Pseudomonas aeruginosa and Acinetobacter baumannii as well. The potential of $E$. coli acquiring KPC is concerning because of its communitywide distribution as a commensal organism.

VIM: Verona Integron-encoded-metallo- $\beta$-lactamase this is a class "B" $\beta$-lactamase first identified in Verona Italy in 1997 in a $P$. aeruginosa clinical isolate. This family has 10 members and is found mostly in P. aeruginosa. VIM-2 is the most reported metallo- $\beta$-lactam world wide.

NDM: New Delhi metallo- $\beta$-lactamase is a class "B" $\beta$-lactamase capable of hydrolyzing penicillins, cephalosporins and carbapenems. The Indian subcontinent is the primary reservoir. This pathogen was first reported in Indian hospitals in 2006, and has since spread globally.

OXA-48: Oxacillin-hydrolyzing- $\beta$-lactamase is a class " $\mathrm{D}$ " serine $\beta$-lactamase, and OXA-48 is the carbapenemase of concern. It was first isolated in Turkey in 2001 from an Enterobacteriacea strain. Most cases have been reported in $K$ pneumoniae and are resistant to all $\beta$-lactams including carbapenemases. Most reports of this strain are in Turkey, North Africa and India, but rarely in the USA.

\section{CRE - Treatment and Carbapenem Antibiotics}

Carbapenem Antibiotics: Of all the different classes of $\beta$-lactamases, the carbapenemases have the broadest activity spectrum and greatest potency against gram-positive and gramnegative, aerobic and anaerobic bacteria. They all have low oral availability and so must be administered parenterally. All carbapenem antibiotics are eliminated by renal excretion [86]. The carbapenems that are available in the USA are: Imipenem (FDA approved 1985; Meropenem (FDA approved 1996); Ertapenem (FDA approved 2001) and Doripenem (FDA approved 2008). In addition, another carbapenem - Biapenem- was approved for use in Japan, China and Korea (2002).

The carbapenems have a safety profile similar to other $\beta$-lactam antibiotics. They play a very important role in fighting infections compared to all other classes of $\beta$-lactam antibiotics. They are often used to treat patients with serious infections as the antibiotic of last resort. However, the emergence of multidrug resistant pathogens threatens to reduce the clinical effectiveness of the carbapenems [30].

Treatment of ESBL infections: ESBLs are primarily produced by the Enterobacteriacea family of Gram-negative organisms, especially from $K$ pneumoniae and $E$. coli. They are also produced by the gram-negative bacteria Acinetobacter baumanii and Pseudomonas aeruginosa. ESBLs are classified as Class A $\beta$-lactamases. They are plasmid mediated enzymes that hydrolyze cephalosporins and monobactams but not cephamycins nor carbapenems. Treatment options are [30,33,34,37]:

- Carbapenem: are the antibiotic of choice against severe ESBL infections. They are rapidly bactericidal and have time dependent killing. They are also effective against other $\beta$-lactamase. They also offer low resistance rates and reduced mortality to patients.

- Fluoroquinolone: is recommended for treatment of urinary tract infections (ESBL)

- Pipericllin- Tazobactam: This is not a first line treatment, and presents lower susceptibility rates for ESBL infections. It is not used for empirical coverage when ESBL rates are high.

- Cefepime: Is a fourth generation cephalosporin. Its use leads to selection for resistant strains, and is less effective than carbapenems. Cefepime is not a first line treatment and is not used as monotherapy. It is used in combination with other antibiotics such as aminoglycosides and fluorquinolones.

- Fosfomycin: While an old drug, it lacks cross resistance with other antibiotics. It has a wide spectrum of activity covering many gram-negative and gram-positive pathogens including ESBL and CRE. It is available in the USA only as an oral formulation and used to treat urinary tract infections. In Europe an intravenous formulation is available.

- SMX - TMP (Sulfamethoxazole - Trimethoprim): This class can be used to treat ESBL infections in patients that are allergic to $\beta$-lactams. However, high resistance rates to ESBL and CRE limit their effectiveness.

- Aminoglycosides: This class can be effective if lab testing shows that isolates are sensitive to aminoglycosides. This class should not be used as mono therapy against ESBL infections.

Treatment options for CRE Infections: Tigecycline, Aminoglycosides, Polymyxin B and Colistin, Fosfomycin,. For treatment of CRE infections and carbapenemase producing infections, these antibiotics can be used as monotherapy or in combination therapy $[30,29,35]$. Table 5 shows the various 
treatment options with these antibiotics - used in both monotherapy and in combination therapy. Because of limited clinical data, the choice of treatment for CPE and CRE infections is controversial. The extensive use of combination therapy remains under debate, as well as the optimal choice of drugs when combinations are used. Most clinical studies have involved patients with KPC and VIM producing strains. Study results are inconsistent, which may be due to different clinical conditions at widely different global locations, as well as variations in patient groups, severity and type of infections and causative bacteria.

\begin{tabular}{|c|c|}
\hline Table 5: Therapeutic Treatment for CRE Infections \\
\hline Monotherapy & Combination Therapy \\
\hline Carbapenems & Colistin + Meropenem \\
\hline Fosfomycin & Colistin + Tigecycline + Meropenem \\
\hline $\begin{array}{c}\text { Gentamycin } \\
\text { Polymyxins (Colistin \& } \\
\text { Polymyxin B) }\end{array}$ & Colistin + Tigecycline \\
\hline Tigecycline & Colistin + Aminoglycosides \\
\hline
\end{tabular}

Table assembled by author based on data from [29]

The treatment options for CRE infections are fewer than for ESBL infections. The primary elements for this application are [28,33]:

- Carbapenems: Using carbapenems as monotherapy is discouraged, as it leads to increased resistance. However carbapenems are an essential element of combination therapy.

- $\quad$ Polymyxins (Colistin \& Polymyxin B): This is an older drug and is active against most gram-negative bacteria and CPE isolates. It is usually used in combination with other agents. Some adverse effects are nephrotoxicity (reversible) and neurotoxicity (rare).

- $\quad$ Tigecycline: It has a broad spectrum of activity against gram-positive and gram-negative bacteria including CPE. Nonsusceptibility to tigecycline against KPC-producing K. pneumoniae is becoming more common in patients who have bene treated with this agent.

- Aminoglycosides: Is effective against KPC-pneumoniae producing strains, and is always used in combination therapy. It is not effective against NDM-producing Enterobacteriacea.

- Fosfomycin: the oral formulation is used to treat urinary tract infections. For systemic infections, intravenous fosfomycin is used in combination with another agent Combinations of carbapenem and fosfomycin are an option for treating CRE strains that are resistant to colistin.

Monotherapy vs Combination Therapy: The preferred clinical practice is to treat invasive infections with a combination of two active agents based on susceptibility patterns of the infection strain. This approach results in lower patient mortality compared to monotherapy treatment. The dosing for each agent should be optimized by using high doses. Uncomplicated urinary tract infections can be managed with a single agent. Clinical data suggests that combination treatment (two or more agents) that are active against the infecting bacterial strain is superior in outcomes compared to monotherapy. It has been shown that combination therapy is more effective against most CPE infections even when the bacteria are resistant to an individual drug [30, 32]. Most combination treatments utilize colistin. It is believed that colistin acts to increase the permeability of the bacterial outer membrane. This effect in turn facilitates action by the other agents used [33]. Clinical studies have also shown that combinations of three antimicrobial agents produce lower mortality rates (i.e.- colistin, tigecycline, carbapenems) [34]. Some clinical studies have shown that prescribing drug combinations for patients with K. pneumoniae-producing VIM and OXA resistant producing infections that contain carbapenems had a significantly lower mortality rate than combination treatment without carbapenems [37].

Empirical Therapy Considerations: For patients suffering from severe septic infections and septic shock, use of empirical antibiotic therapy should cover all of the patient's suspected bacterial infections. Empirical treatment with colistin and carbapenems or aminoglycosides is justified for treating severely ill patients with a suspected CRE infections. However, exclusive use of a broad spectrum antibiotic should be avoided to prevent further selection of resistant bacteria and also prevent fungal super infections and C. difficile outbreaks in hospitals [34,38].

\section{Risk Factors for Development and Spread of CRE}

General Risk Factors: CREs are easily introduced because they are highly transmissible, resulting in colonization or infection of patients. Dissemination of mobile genetic elements coding for resistance and especially with multidrug resistant strains has been the cause of many reported outbreaks in hospitals [39]. The European Center for Disease Prevention has listed general risk factors associated with colonization or infection with CRE, including: the length of hospitalization (time at risk); severity of illness; mechanical ventilation; admission to the ICU, presence of wounds, positive blood culture, prior surgery, transfer between hospital units, prior hospital stay, presence of catheters and intubation [39].

Prior Antimicrobial Use: Prior antibiotic exposure has been found to be a risk factor for colonization and infection with CRE and especially for K. pneumoniae (KPC) [39,30].

- $\quad$ Carbapenems: Prior use of carbapenems is identified as an independent risk factor for the acquisition of KPC producing $K$ pneumoniae and for carbapenem resistant KPC and carbapenem resistant E.coli [40]. A study done at a Chicago hospital in 2013 demonstrated that increasing carbapenem use was directly correlated with increasing resistance in Enterobacteriaceae [41].

- Cephalosporins: Prior use of an extended spectrum cephalosporin is identified as a risk factor for the acquisition of KPC producing K. pneumoniae, and also as an independent risk factor for the acquisition of KPC producing K. pneumoniae for carbapenemase resistant K. pneumoniae and also for carbapenem resistant E.coli. 
- Fluorquinolones: Prior use of a fluoroquinolone antibiotic is identified as a risk factor for the acquisition of KPC producing Klebsiella pneumoniae. Use of fluoroquinolones is also an independent risk factor for the acquisition of carbapenemase resistant $K$. pneumoniae.

- $\quad$ Other antibiotics: Other antibiotics associated with risk of acquiring carbapenemase resistant enterobacteriacea are the anti pseudomonal penicillins and metronidazole.

Cross Border Transmission \& Patient, mobility: Cross border transfer of patients is a documented risk factor for the introduction of carbapenemase producing Enterobacteriaceae into healthcare settings and systems. When patients are infected or colonized with carbapenemase-producing Enterobacteriaceae are transferred across borders, the risk of CRE being introduced and spread into healthcare facilities in the country of destination is increased. The risk is higher when patients are transferred from areas with high rates of CRE to healthcare facilities in another country, or if such patents have received medical care abroad in areas with high rates of CRE [39]. Cross-border transmission has been reported in Asia, Europe and North America involving carbapenemase producing K. pneumoniae. For example, it has been reported that CRE K. pneumoniae was reported in patients in hospitals in Norway and Finland from patients that were previously hospitalized in Greece. All isolates belonged to a clonally related K. pneumoniae strain from Greece [42].

Infection Control Measures: Carbapenemase-producing Enterobacteriaceae can colonize and infect not only patients who are debilitated, immune-compromised or critically ill, but also those patients that were previously healthy and became colonized or infected in healthcare settings that practice poor infection control [39]. CREs and other ESBL producing organisms can easily spread within the hospital environment. Preventing spread of these organisms from patient to patient is the main focus of infection control. A major issue is hand hygiene for healthcare professionals. The cleaning of medical equipment and prevention of colonization of the hospital environment are also important infection control measures. It is important to screen patients being admitted or transferred from other institutions including from nursing homes. Surveillance of infected and high risk patients is an important action for monitoring an outbreak and also to prevent one [39]. Small hospital outbreaks tend to be caused by a single clone and usually occur in high risk areas such as the ICU, neonatal units and hematology-oncology units. Large outbreaks usually involve several circulating strains of organisms at one time and in several different areas of a healthcare setting [43].

The prevention of the spread of carbapenemase producing pathogens relies on early detection [44]. Patients who undergo screening should include: (i) patients who were hospitalized while abroad and then transferred to another country; (ii) general risk-intensive care patients, immunocompromised patients). Screened patients should be kept in strict isolation before obtaining results of the screening (at least 24 to 48 hours). Because the reservoir of carbapenemase producers remains in the intestinal flora, use of rectal swabs for screening are adequate to perform this screening measure- and then plated directly onto screening media. After this screening procedure, carbapenemase producers may be identified through a variety of techniques including antibacterial drug susceptibility testing and molecular \& PCR based techniques [22,29].

\section{CRE - Extent of the Problem (USA and International)}

Increasing Global Prevalence of CRE: The first Carbapenemase-resistant Enterobacteriacea (CRE)- $K$. pneumoniae was identified in North Carolina in the 1990s. Since then, CRE have spread globally and are endemic in some countries, including USA, Italy, Greece, Israel, China and India among others. Infections with CRE are being increasingly reported from healthcare facilities in the developed world, and with a higher prevalence over the past five years. Moreover, the presence of CRE infections are being increasingly reported in LMI counties as well. For example, in U.S. hospitals $11 \%$ of $K$. pneumoniae and $2 \%$ of $E$. coli were resistant to carbapenems in 2013. As a comparison, in India $13 \%$ of $E$. coli were resistant to carbapenems in 2012 and $57 \%$ of K pneumoniae were resistant to carbapenems in 2014 [36,45].

Global Reporting of Extended Spectrum $\beta$-Lactamase (ESBL) Producing Strains: ESBLs can inactivate all penicillins and cephalosporins, including third generation cephalosporins and monobactams. In Europe, 17 of 22 countries reported that $85 \%$ to $100 \%$ of E.coli isolates were ESBL positive. In the USA, healthcare-associated ESBL-producing Enterobacteriaceae made up $14 \%$ of E. coli isolates and $23 \%$ of $K$. pneumoniae isolates. ESBL-producing Enterobacteriaceae are increasing in Asia. In China in 2011, ESBL-producing E. coli accounted for $71 \%$ of E. coli isolates, and more than $50 \%$ of $K$. pneumoniae strains produced ESBL. In Latin America, ESBL-producing Enterobacteriaceae prevalence is rising. Rates of ESBL in E. coli in Mexico were as high as $41 \%$ in 2009 . In 2014, resistance of $K$. pneumoniae isolates to third-generation cephalosporins ranged from 19\% in Peru to $87 \%$ in Bolivia. In North Africa, ESBL prevalence ranged from 12 to $99 \%$ in hospitals and from 1 to $11 \%$ in communities. In East Africa, ESBLs were found in 38 to $63 \%$ of hospital samples and in $6 \%$ of community samples. In Central Africa, 55\% to $83 \%$ of hospital samples and 11 to $17 \%$ of community samples were ESBL positive [46].

CRE Prevalence in Europe: A multi country survey was carried out in Europe in 2012 with 33 countries reporting data [47]. The prevalence of CRE is variable across Europe, but is higher in Greece and Italy and lower in the Nordic countries. It was found that $K$ pneumoniae was the most prevalent type of Enterobacteriaceae species harboring CRE in those countries. The five most common CRE among Enterobacteriaceae reported by these countries are the metallo- $\beta$-lactamases IMP, VIM, NDMfrom Amber Molecular Class B; OXA-48 and its derivatives from Amber Molecular Class D; KPC from Molecular Class A [29]. Overall, KPC producing Enterobacteriacea are the most frequently detected among CRE in Europe. However, rising 
numbers of OXA-48 producing isolates are being reported in Europe. OXA-48 is the most frequently detected CRE in Belgium, France, Malta and Spain. Most of the OXA-48 producing strains observed in France were associated with travel to and/or hospitalization in North Africa where OXA-48- producing strains are endemic [47]. The major concern with OXA carbapenemases is their ability to rapidly mutate and expand their spectrum of activity. In the United States, $>50 \%$ of $A$. baumannii are resistant to carbapenems due to production of OXA class carbapenemases [48]. It appears that prevalence of CRAb in Europe is under reported. This is because surveillance and reporting of CRAb are not performed routinely and there are fewer reference laboratories for CRAb in Europe. A survey done in 2011 -2012 of HAI and antimicrobial use covered more than 1,000 hospitals in 30 European countries [49]. The survey showed that while Acinetobacter species were only associated with $3.6 \%$ of all microbial species identified in Healthcare Acquired Infections (HAI), fully $81.2 \%$ of all Acinetobacter isolates were reported to be resistant to carbapenems. In comparison, whereas E.coli and Klebsiella species together accounted for $24.6 \%$ of all microbes isolated in HAI, only $9.3 \%$ of those isolates were resistant to carbapenems.

\section{CRE - Problem: Hospital Overuse of Antibiotics}

General Considerations: There is a causal association between use of antimicrobial drugs and the emergence of AMR. Changes in antibiotic use are also paralleled by changes in the prevalence of resistance. AMR is more prevalent in healthcare associated bacterial infections than with community acquired infections. Patients with healthcare associated infections caused by resistant strains are more likely than control patients to have received prior antibiotic treatment within the hospital. Those patients with the highest rates of resistant strains also have the longest duration of exposure to antibiotics. The longer exposure period increases the likelihood of colonization with resistant organisms $[13,30,41]$.

Overuse of Broad Spectrum Antibiotics: It is the practice in hospitals to give patients broad spectrum antibiotics, even when a specific pathogen is identified. Such practices contribute to the spread of resistant strains to many non- target organisms. In a survey of 605 hospitals in the USA during 2009/2010, only 59\% of patients received appropriate antibiotic treatment [50]. In this survey, during the 5 th day of therapy, $6 \%$ of antibiotic treatments regimens were unchanged despite there being negative bacterial cultures in $58 \%$ of the patients on therapy.

The survey indicated that broad spectrum antibiotics were commonly prescribed to patients even when the signs of infection were not present - including negative cultures -and the antibiotic treatment was not discontinued. Another survey done in 2010 of 323 hospitals in the USA $56 \%$ of patients received an antibiotic during their hospital stay, and primarily with broad spectrum antibiotics [50]. Among the patients receiving antibiotics, $37 \%$ of antibiotic treatments given were judged as needing improvement. The use of diagnostic tests in these cases would have resulted in more appropriate antibiotic regimen to those patients. The rates of inappropriate prescribing of antibiotics is also common in international hospitals. In hospitals in Nepal, the rates of inappropriate prescribing of antibiotics is in the range of 10 to $42 \%$. In Vietnam, it was found that $1 / 2$ of hospital prescriptions for antibiotics were inappropriate [49].

Antibiotic Use for Surgery Prophylaxis: In high income countries, pre surgical antibiotics are standard treatment to prevent post surgical infection. However, in many LMICs (Low \& Middle Income countries) antibiotics are commonly given after surgical procedures. This practice presents a higher risk of surgical site infections. It is found that LMIC hospitals used seven times more antibiotics when they are given post surgery rather than pre surgery [49]. This practice increases costs and also increases AMR potential. Even when antibiotics are given before surgery, the regimen or duration of the treatment may be suboptimal. A survey of hospitals in India showed a range of 19\% to $86 \%$ of patients received inappropriate antibiotic prophylaxis [51]. Proper antibiotic prophylaxis improves both hygiene, better surgical techniques and lowers the rate of surgical site infections.

Suboptimal Use of Antibiotics in Hospitals: The suboptimal use of broad spectrum antibiotics and also suboptimal use of post surgical antibiotics is prevalent both in North America, Europe and the LMIC, and so represents an opportunity to improve antibiotic use in hospital settings. Estimates have been made that a range of $20 \%$ to $50 \%$ of total antibiotic use in hospital settings are inappropriate $[50,52]$. Inappropriate antibiotic use includes: (i) Use of antibiotics when there is no benefit from its use, such as treating urinary tract infections caused by virus and (ii) incorrect antibiotic selection, incorrect dosage, incorrect duration of treatment the patient receives.

\section{CRE - Problem: Outpatient and Nursing Home Overuse of Antibiotics}

Antibiotic Use in the Community: Overuse and misuse of antibiotics in outpatient settings is a major driver of AMR. An estimated $80 \%$ of all antibiotics are consumed outside of hospitals in outpatient settings [49]. These healthcare settings include: i) self medication; (ii) outpatient clinics and private physician offices; (iii) nursing homes.

Antibiotic Overuse by Self Medication: Outpatient use also includes antibiotics purchased by consumers directly and without a prescription. Although most countries require a prescription as condition for purchase of an antibiotic, these regulations are not enforced in most LMIC, or do not exist. Nonprescription use of antibiotics can range from $19 \%$ to over $90 \%$ outside of the USA and Europe. For example, in rural and urban pharmacies in Vietnam, $88 \%$ to $91 \%$ of all antibiotic sales in a survey made were without a prescription. Similarly, most antibiotics sales in Saudi Arabia (78\%) and Syria (87\% to 97\%) were dispensed without a prescription [49]. In addition, people in both developed and developing countries are increasingly purchasing antibiotics from internet sources and which are difficult to control. Most self medication uses are for treating common colds and upper respiratory tract infections- which are mostly of viral origin and not responsive to antibiotics. 
Antibiotic Overuse by Prescribers: Healthcare providers also play a role in driving inappropriate antibiotic use in the community. They routinely prescribe antibiotics for infections that are not caused by bacteria. A major factor for over prescribing are the pressure that patients put onto prescribers for an antibiotic prescription. In a survey done in the USA, $48 \%$ of respondents indicated that they expected an antibiotic when they visited a doctor [49]. In a survey done in France, $50 \%$ of interviewees expected an antibiotic for treatment of influenza like illness [49]. Other factors leading to high rates of antibiotic prescriptions by prescribers include diagnostic uncertainty. Since most diagnostic lab methods are based on the culture of pathogens that require 36 to 48 hours to provide results, few infections are accurately diagnosed in these practice settings. In the absence of a clear diagnosis, the prescriber often feels pressured to prescribe antibiotics to be on the safe side or to prevent secondary bacterial infections $[49,53]$. A recent study commissioned by the Pew Charitable Trusts in May 2016 found that $13 \%$ of all out patient office visits in the USA (154 million visits/year) resulted in an antibiotic prescription [54]. About 31\% of these antibiotic prescriptions (47 million) are considered to be unnecessary. The Pew study also found that $44 \%$ of outpatient antibiotic prescriptions (68 million) were written to treat patients with acute respiratory conditions (including: sinus infections, middle ear infections, pharyngitis, viral upper respiratory tract infections -common cold; bronchitis; bronchiolitis; asthma; allergies; influenza and pneumonia). Half of these prescriptions are unnecessary because many of these conditions are viral illnesses or other conditions that do not respond to antibiotics. In addition to using medications that will not affect the illness, such improper use of antibiotics also carries the risk of adverse drug effects and other side effects. It is estimated that improper use of antibiotics in the USA results in 140,000 emergency room visits per year [54]. In May 2015, the White House released a national action plan for combatting antibiotic resistance. This plan has the goal of reducing inappropriate outpatient antibiotic use by $50 \%$ by 2020 [55-57]. Based on this estimate of unnecessary prescribing, the White House goal can be reached by reducing outpatient antibiotic use by $15 \%$ overall, which would result in about 23 million fewer antibiotic prescriptions by 2020 .

Antibiotic Overuse in Nursing Homes: In the USA, 1.6 million people live in nursing homes. Out of that population, about 250,000 people will acquire infections every year. And out of that population, nursing home residents will acquire 27,000 antibiotic resistant infections. Itis further estimated that up to $70 \%$ of nursing home residents are prescribed an antibiotic every year. And of these antibiotic prescriptions, from $40 \%$ to $75 \%$ is prescribed incorrectly-ether being unnecessary or else incorrectly for the drug prescribed, or the dose, or the duration of therapy [58]. Of particular concern is the widespread use of broad spectrum oral antibiotics such as quinolones for which overuse can be a major driver of AMR. Nursing home residents are more vulnerable to infections because of biological factors (reduced immune system, prevalence of chronic diseases, use of invasive devices such as urinary catheters and feeding tubes) and environmental (crowding, sanitation issues).Nursing homes are being increasingly identified as important reservoirs for the development of Multidrug-Resistant (MDR) organisms and their transmission into the community. The three most frequently reported infections in nursing homes are: Urinary Tract Infections (UTI), Respiratory Tract Infections (RTI) and Skin and Soft Tissue Infections (SSTI) [58]. This same group of infections are the leading causes for antibiotic prescribing in nursing homes. Some studies also report that UTIs and RTIs are the most commonly observed causes for hospital admissions among the elderly from nursing homes. Overuse of antibiotics in nursing homes not only produces UTI and RTI that are resistance to antibiotic treatment, but are also a major cause of hospital admissions of nursing home residents. Studies have demonstrated that nursing homes are a reservoir for carriage of three major groups of MDR organisms (i.e. MRSA, VRE and MDR GNB (Gram-negative bacteria). However in recent years there has been a shift towards greater colonization with MDR GNB. Some studies show MDR GNB colonization far exceeding that of MRSA and VRE. The emergence of MDR GNB in nursing homes goes beyond asymptomatic carriage of these organisms; there has been a regular increase of MDR GNB clinical isolates as well [59]. It has been found that prior exposure to antibiotics is a prominent risk factor associating with both colonization and infection of both MDR gram-positive and gram-negative organisms $[39,41,58]$. Several studies have described the inappropriate and excessive use of antimicrobials has led to the development of AMR in nursing homes. Moreover, repeated antimicrobial utilization, and particularly the repeated use of broad-spectrum antibiotics will increase the risk of Clostridium difficile infection-a leading cause of hospitalization of nursing home residents [58]. Reduction of unnecessary use of antibiotics in nursing homes can be an effective strategy to curb the rapid emergence of MDR organisms in this healthcare setting $[58,60,62]$.

Areas of Potential Antibiotic Misuse in Nursing Homes: Major areas of potential antibiotic misuse in nursing home that lead to development of AMR are listed below:

1. Prophylactic antibiotics for UTI: There is little evidence to support the use of long-term urinary prophylaxis. However, there is strong evidence showing that prolonged antibiotic use in the absence of infection will always select for resistant organisms.

2. Empiric prescribing without microbiological investigation: Causative etiologic agents should be identified through microbiological testing, especially for symptomatic UTIs, to guide the adjustment of empiric antibiotic therapy.

3. Treatment of asymptomatic bacteriuria: This condition is common with chronically catherized patients. However, antibiotic treatment will not prevent recurring bacteriuria or symptomatic infections. The right strategy is to change indwelling catheters prior to starting antibiotic therapy and taking a urine sample collected from the newly placed catheter. Discontinuation of catheter use and proper aseptic technique on catheter changing are the keys to preventing UTIs and other urinary complications [58]. 
4. Widespread prescribing for upper RTIs or acute bronchitis: Among elderly nursing home residents, upper RTIs are usually caused by viral pathogens. Therefore, empiric antibiotic treatment is both unnecessary and ineffective. It is necessary to differentiate between bacterial or viral origin to reduce inappropriate use of ABS to treat RTS

5. Prolonged duration of antibiotic treatment: It is generally considered that antibiotic courses of 7 days or less are as effective as longer treatment duration for the majority of common bacterial infections. In contrast, unnecessary prolonged antibiotic treatment increases patient risk for side effects and AMR.

6. Widespread prescribing of quinolones as empiric treatment for UTIs: The quinolone antibiotics are frequently used to treat both low and complicated UTI because of their high bioavailability, long half-life, and broad-spectrum activity spectrum. Consequently a high rate of quinolone-resistant gram negative organisms is often observed in nursing homes with a high use of quinolones $[58,61,63]$.

\section{CRE - Solution: Prudent Use of Antibiotics in Healthcare}

General Considerations:The consensus of experts is that prior use of all antimicrobial products, and more specifically the carbapenems, 3rd and 4th generation cephalosporins and fluorquinolones increases the risk of infection or colonization with CRE [64-73] (Table 6). High rates of multi drug resistant organisms (MDROs, ESBL producing bacteria are considered to be independent risk factors for the spread of carbapenemase resistant mechanisms. Experts suggest that no particular class of antibiotic is the predominant factor for the selection of carbapenemase producers. Rather, it is the intensity and duration of antibiotic treatment which are the most important variables for the selection of CRE producing bacteria [74]. A review of the medical literature indicates there is an important relationship between prior antimicrobial therapy and the subsequent identification of carbapenemase-producing bacteria. In a four year case control study of 102 patients, the only covariate independently associated with CRE in all multivariate analyses

Table 6: Antibiotic Usage in U.S. Hospitals (2006 to 2012) DOT / 1000 PD (i)

\begin{tabular}{|c|c|c|c|c|c|c|c|c|c|}
\hline Antibiotic Class & 2006 & 2007 & 2008 & 2009 & 2010 & 2011 & 2012 & Change 2006 to & $2012(\%)$ \\
\hline All (ii) & 53.8 & 54 & 54.9 & 55.7 & 55.7 & 56.4 & 55.6 & +2.8 & \\
\hline $1^{\text {st }} \& 2^{\text {nd }}$ Generation Cephalosporins & 20.4 & 20.3 & 20.1 & 20.2 & 20.1 & 19.5 & 18.9 & -7.4 & \\
\hline $3^{\text {rd }} \& 4^{\text {th }}$ Generation Cephalosporins & 10.9 & 10.9 & 11.1 & 11.6 & 12.1 & 13.3 & 13.4 & +32.1 & \\
\hline Fluoroquinolones & 16.8 & 16.7 & 16.9 & 16.4 & 15.8 & 15.7 & 15 & -10.7 & \\
\hline Glycopeptides & 8.2 & 8.9 & 7.9 & 10.7 & 11.3 & 12.3 & 12.9 & +57.3 & \\
\hline B-Lactamase Inhibitors & 7.5 & 8 & 8.6 & 9.1 & 9.5 & 10.2 & 10.4 & +38.7 & \\
\hline Carbapenems (iii) & 1.7 & 2 & 2.3 & 2.6 & 2.7 & 2.9 & 3 & +76.5 & \\
\hline Penicillins & 6 & 5.7 & 5.5 & 5.1 & 5.2 & 5.3 & 5.3 & -11.7 & \\
\hline
\end{tabular}

Notes: (i) DOT = Days of Therapy; PD = Patient Days

(ii) Overall use of antibiotics from 2006 to $2012=2.8 \%$ increase

(iii) Use of carbapenem antibiotics from 2006 to $2012=76.5 \%$ increase

was the cumulative number of prior antibiotic exposures [74,75]. In another case-control study performed in Greece and lasting 26 months (96 ESBL-carbapenem-resistant K. pneumoniae and 55 ESBL-carbapenem-sensitive $K$. pneumoniae) identified the key risks factors as: (i) prior cumulative exposure to antibiotics and (ii) increasing duration of prior antibiotic treatment [74,39]. The antibiotic treatments covered in these studies included use of a $\beta$-lactam or $\beta$-lactamase inhibitor or a combination of fluoroquinolone and carbapenem. The results of these studies in Greece are consistent with previous reports that no particular class of antibiotic is the predominant predisposing factor for selection of carbapenemase production. However, the most important variables responsible for selection of carbapenemase -producing bacteria are the intensity and duration of antibiotic therapy [74]. The evolution of CREs follows a pathway driven by the misuse and overuse of several generations of $\beta$-lactam antibiotics over the last 40 to 50 years. Controlling the spread of CRE infections requires both the prudent use of antibiotics plus infection control. Additional clinical studies suggest that the patient's cumulative exposure history is likely to be a greater risk factor than any one specific antibiotic exposure to determine a patient's chance of acquiring a resistant pathogen [39] (Table 7).

Reducing Overuse of Antibiotics: From 20\% to 50\% of all antibiotic use is estimated to be inappropriate, covering both (i) the use of antibiotics when there is no possible health benefit, such as treating upper respiratory tract infections caused by virus origin, and (ii) the suboptimal use of antibiotics such as incorrect choice of prescribed drug (i.e.- unnecessary broad spectrum antibiotic), incorrect dosage or duration, or poor patient adherence, substandard quality of antibiotic product - all of these factors contribute to the development and spread of AMR. It has been found in Europe that regional and national campaigns to educate healthcare workers and patients about the danger of AMR can help change behavior to reduce inappropriate 


\begin{tabular}{|c|c|c|c|}
\hline Country & Sales 2005 (i) & Sales 2010 (i) & $\begin{array}{c}\text { \% Sales Increase } \\
2005-2010\end{array}$ \\
\hline Netherlands & 9.5 & 18.5 & 95 \\
\hline USA & 18 & 21 & 17 \\
\hline Brazil & 2.5 & 4 & 60 \\
\hline Vietnam & 1 & 9 & 800 \\
\hline Indonesia & 3.5 & 10 & 186 \\
\hline India & 11 & 63.5 & 477 \\
\hline Pakistan & 37 & 90 & 143 \\
\hline Egypt & 24.5 & 82 & 237 \\
\hline
\end{tabular}

Notes: (i) Retail sales are expressed as Standard Units (SU) per 1,000 population .

Table assembled by author from data in [13].

prescribing [75]. Two such effective campaigns took place in Belgium and France [49]. Prior to this national education/ awareness campaign, both countries had the highest rate of antibiotic consumption in Europe. The French campaign started in 2001 and achieved a reduction of $27 \%$ for antibiotic prescribing over five years. The Belgian national media campaign achieved a $36 \%$ reduction in antibiotic prescriptions over a seven year period. Both countries also showed a corresponding reduction in antibiotic resistant pneumococci following these campaigns.

\section{Prudent Use Recommendations:}

- $\quad$ Rapid Diagnostics: Patients with sepsis must be treated quickly, but empirical antibiotic treatment is often started before reliable microbiology assessments are available. This results in some patients receiving incorrect antibiotic treatment. More rapid diagnostics are needed so that the appropriate antibiotic treatment can be administered more quickly and using broad spectrum antibiotics for the shortest duration to reduce selection pressure for resistance.

- Colonizaton Prevention: The prevention of colonization can have considerable benefit in the era of CRE infections. Hospitals should take rectal samples of patients at risk. If the isolates are KPC positive, the patient should be isolated in a single room and bathed daily with chlorhexidine gluconate.

- Heterogeneity of Antibiotic Usage: Patterns of antibiotic use in which the same antibiotic given is given repeatedly (homogeneity) is associated with higher rates of resistance than when there is variability in antibiotics prescribed for the patients (heterogeneity). It has been shown that monthly cycling of four antibiotics (PTZ, imipenem/cilistin, ceftazidime, ciprofloxacin) as the primary antibiotic to treat suspected Gram-negative infections was associated with an overall improvement in the antibiotic susceptibility profile of Gram -negative organisms compared with the medical ICU in the same hospital where cycling was not performed. These results suggest that increased diversity of prescribing may correlate with reduced levels of resistance [39].
Table 8: WHO List of Essential Medicines: Antibiotics (20 $0^{\text {th }}$ List March 2017) WHO has devised a list for antibiotic utilization to support antibiotic stewardship activity and reduce antimicrobial resistance on a global basis. There are three different categories: ACCESS, WATCH and RESERVE groups KEY ACCESS ANTIBIOTICS

Beta-lactam Antibiotics

\begin{tabular}{|c|c|c|}
\hline Amoxicillin & Cefalexin & Cloxacillin \\
\hline Amoxicillin + Clavulanic Acid & Cefazolin & Phenoxymethyl Penicillin \\
\hline Ampicllin & Cefixime $^{*}$ & Piperacillin + Tazobactam $^{*}$ \\
\hline Benzathine Benzylpenicillin & Cefotaxime $^{*}$ & Procaine Benzyl Penicillin \\
\hline Benzylpenicillin & Ceftriaxone $^{*}$ & Meropenem \\
\hline \hline
\end{tabular}

\begin{tabular}{|c|c|c|}
\hline \hline Other Antibiotics & \multicolumn{2}{|l|}{} \\
\hline Amikacin & Clindamycin & Spectinomycin \\
\hline Azithromycin & Doxycycline & $\begin{array}{c}\text { Sulfamethpxazole + } \\
\text { Trimethoprim }\end{array}$ \\
\hline Chloramphenicol & Gentamicin & Vancomycin (oral) $^{*}$ \\
\hline Ciprofloxacin $^{*}$ & Metronidazole & Vancomycin (parenteral)* $^{*}$ \\
\hline Clarithromycin $^{*}$ & Nitrofurantoin & \\
\hline
\end{tabular}

Antibiotics that are first or second choice in at least one of the reviewed syndromes, and are antibiotics that shoul be widely available, affordable and quality assured. Selected ACCESS antibtioics $\left({ }^{*}\right)$ may also be included in the WATCH group.

\section{Watch Group Antibiotics}

Quinolones \& Fluoroquinolones: (Ciprofloxacin, Levofloxacin, Moxifloxacin, Norfloxacin

3rd Generation Cephalosporins: (Cefixime, Ceftriaxone, Cefotaxime,

Ceftazidime

Macrolides: (Azithromycin, Clarithromycin, Erythromycin)

Glycopeptides: (Teicoplanin, Vancomycin)

Antipseudomonal Penicillins + Beta Lactam Inhibitor: (Piperacillin-

Tazobactam)

Carbapenems: (Meropenem, Imipenem, Cilastin)

Penems: (Faropenem)

Antibiotic classes that have higher resistance potential and recommended as first or second choice treatments only for specific and limited number of indications. These antibiotics are key priorities for antibiotic steward programs and are at high risk of selection for bacterial resistance.

\section{Reserve Group Antibiotics}

\begin{tabular}{|l|l|}
\hline Aztreonam & Fosfomycin (IV) \\
\hline $\begin{array}{l}\mathbf{4}^{\text {th }} \text { generation cephalosporins } \\
\text { (Cefepime) }\end{array}$ & Oxazolidinones (Linezsolid) \\
\hline $\begin{array}{l}\mathbf{5}^{\text {th }} \text { generation cephalosporins } \\
\text { (Ceftaroline) }\end{array}$ & Tigecycline \\
\hline Polymixins (Polymixin B, Colistin) & Daptomycin \\
\hline
\end{tabular}

Polymixins (Polymixin B, Colistin) Daptomycin

Antibiotics that are used as "Last Resort" options - for highly specific patients and settings when all alternative antibiotics have failed

Ref: WHO Medicines Website (March 2017)

http:/www.who.int/medicines/publications/essentialmedicines/en

Citation: Kardos N (2017) Overuse of Antibiotics and Antibiotic Resistance in Medical Applications Featuring CarbapenemasePage 15 of 21 Resistant Enterobacteriaceae (CRE). SOJ Microbiol Infect Dis 5(5):1-21. 
WHO Essential Medicines List Update (Antibiotics): In March 2017 WHO updated its Essential Medicines List, the largest revision of WHO guidelines for use of antibiotics in its 40 year history $[42,76]$. The revised WHO list is a primary guideline for prudent use of antibiotics and is intended to assist antibiotic stewardship and support local and national guidelines for rational use. WHO has grouped antibiotics into three separate categories. The first category is 'Access' covering common clinical infections (i.e. Urinary tract infections, Pneumonia) such as amoxicillin [76]. The Access antibiotics are narrow spectrum and which have less likelihood of producing resistance. The great majority of antibiotics prescribing for common clinical infections should be used from the Access group of antibiotics (Table 8). The second category is 'Watch' covering antibiotics which have a greater chance of producing resistance. The Watch antibiotics are not first line choices for prescribers and should be conserved where possible [76].The third category is 'Reserve' considered as the antibiotics of last resort reserved for specific resistant infections $[42,76]$. Use of the antibiotics on the Reserve list is rising on a global basis because of increasing prevalence of antibiotic resistance [42].

The Avoidable Costs of Antibiotic Misuse:In addition to the which have a greater chance of producing resistance. The Watch antibiotics are not first line choices for prescribers and should be conserved where possible [76].The third category is 'Reserve' considered as the antibiotics of last resort reserved for specific resistant infections $[42,76]$. Use of the antibiotics on the Reserve list is rising on a global basis because of increasing prevalence of antibiotic resistance [42].

The Avoidable Costs of Antibiotic Misuse:In addition to the rise of Antimicrobial Resistance (AMR), the misuse of antibiotics for medical applications represents large avoidable healthcare costs in the USA \$35 billion/year [77]. Within that amount, most of that amount ( $\$ 23$ billion) is incurred through inpatient care (hospital use). The avoidable costs are defined as the added cost of treating a patient with an antibiotic resistant infection relative to a patient with an antibiotic susceptible infection. The estimated avoidable costs for the inpatient setting include longer medical treatments, expensive second-and third-line antibiotic therapies, and screening and diagnostics to detect and prevent the spread of bacterial strains. The cost of excessive antibiotics prescribed in outpatient settings in the USA is $\$ 1$ billion/year [77].

\section{CRE - Solution: Antibiotic Stewardship Programs}

Basis for Antimicrobial Stewardship (AMS) program: An AMS program is a set of coordinated strategies to optimize the use of antimicrobial medications in order to improve patient safety and outcomes, decrease and prevent the development of antimicrobial resistance, and decrease costs [78]. Recent data suggests that up to $55 \%$ of all hospital patients in the USA receive an antibiotic, and that between $30 \%$ to $50 \%$ of the prescribed antibiotics may not be appropriate [79]. The inappropriate prescribing of antibiotics leads to potential complications and adverse drug events. It also may cause hospital acquired infections. Comprehensive AMS programs have been shown to decrease antibiotic use by $22 \%$ to $36 \%$ and halt the development and spread of antimicrobial resistant bacteria [79]. Core members of a multidisciplinary AMS team may include: (i) Infectious disease physician; (ii) Clinical pharmacist with infectious disease training; (iii) Clinical microbiologist; (iv) Information system specialist; (v) Infection control professional; and (vi) Hospital epidemiologist. The CDC reported that in 2014 nationally, 39.2\% of all hospitals had AMS programs (1,642 out of 4,184 hospitals). The national goal is for $100 \%$ of hospitals to have an AMS program by 2020 . The Centers for Medicare and Medicaid Services (CMS) will require hospitals to reduce antibiotic use starting in January 2017 [80].

Core Strategies for an AMS Program: There are two core strategies that provide the foundation for an AMS program. These strategies ae not mutually exclusive [81].

(A) Prospective audit with intervention and feedback strategy: This strategy involves a prospective audit of antimicrobial use with direct interaction and feedback to the prescriber, performed by either an infectious disease physician or a clinical pharmacist. This strategy can result in both reduced inappropriate use of antibiotics and also the improved use of antibiotics. Effective auditing with intervention and feedback can be facilitated through computer surveillance of antimicrobial use.

(B) Formulary restriction and preauthorization strategy: Formulary and preauthorization requirements can lead to immediate and significant reductions in antimicrobial use and cost, and may be beneficial as part of a multifaceted response to a nosocomial outbreak of infection. The use of preauthorization requirements as a means of controlling AMR is less clear, because a long-term beneficial impact on resistance has not yet been established. And in some circumstances, this policy may just shift to using an alternative agent with resulting increased resistance. Preauthorization use policy requires monitoring overall trends in antibiotic use in order to assess and respond to such shifts in use. The effectiveness of a preauthorization process depends on who is making the recommendations. One examples is a study done in a hospital that experienced an increasing incidence of cephalosporin resistant Klebsiella [83]. A preapproval policy was implemented for cephalosporins, resulting in an $80 \%$ reduction in hospital-wide cephalosporin use and a subsequent $44 \%$ reduction in the incidence of ceftazidime-resistant Klebsiella throughout the medical center.

CDC's Core Elements of Antibiotic Stewardship Programs: In 2014, CDC recommended that all acute care hospitals implement AMS programs.The CDC published seven core elements of successful hospital antibiotic stewardship programs. These elements include the pharmacist's role in antibiotic stewardship [84]. The CDC core elements are the following:

1. Leadership Commitment: Dedicating necessary human, financial and information

2. Accountability: Appointing a single leader responsible for program outcomes. Experience with a successful programs show that a physician leader is effective. 
3. Drug Expertise: Appointing a single pharmacist leader responsible for working to improve antibiotic use.

4. Action: Implementing at least one recommended action, such as systemic evaluation of ongoing treatment need after a set period of initial treatment (i;e; "antibiotic time out" after 48 hours)

5. Tracking: Monitoring antibiotic prescribing and resistance patterns

6. Reporting: Regular reporting information on antibiotic use and resistance to doctors, nurses and relevant staff

7. Education: Educating clinicians about resistance and optimal prescribing.

Supplementary Elements of an AMS Program: The Infectious Disease Society of America (IDSA) and the Society for Healthcare Epidemiology of America have also recommend supplemental strategies [81]:

-Antimicrobial cycling and scheduled antimicrobial switch: Antimicrobial cycling- refers to the scheduled removal and substitution of a specific antimicrobial or antimicrobial class to prevent or reverse the development of AMR within an institution. In true cycling, there is a return to the original antibiotic after a defined time as opposed to a simple switch of antibiotics. Antimicrobial cycling is an attempt at controlled heterogeneity of antimicrobial use to minimize antimicrobial selection pressures. There is insufficient data to recommend the routine use of antimicrobial cycling as a means of preventing or reducing AMR over a prolonged period of time. Substituting one antibiotic for another may transiently decrease selection pressure and reduce resistance to the restricted agent. However unless the resistance determinant has been eliminated from the bacterial population, the reintroduction of the original antibiotic will likely select for the expression of the resistance determinant in the exposed bacterial population.

- Combination therapy-Prevention of Resistance Versus Redundant Antimicrobial Coverage: The rationale for combination therapy includes broad spectrum empirical therapy for serious infections, improved clinical outcomes and the prevention of resistance. These recommended situations include the use of empirical therapy for critically ill patients at risk of infection with multidrug resistant pathogens in order to increase the breadth of coverage and the likelihood of adequate initial therapy. However in many situations, combination therapy is redundant and unnecessary, and there is insufficient data to recommend the routine use of combination therapy to prevent the emergence of resistance.

- Streamlining or de-escalation of therapy: Efforts to optimize empirical initial antimicrobial therapy may conflict with good AMS to promote judicious use of antibiotics, because continuing excessively broad therapy contributes to the selection of AMR pathogens. This conflict can be resolved when culture results become available by streamlining or de-escalating antimicrobial therapy to more targeted therapy that decreases antimicrobial exposure and contains cost. The elimination of redundant combination therapy can result in reduced antimicrobial exposure and resistance.

- Dose Optimization: Optimization of antimicrobial dosing based on individual patient characteristics, causative organism, site of infection, and pharmacokinetic and pharmacodynamics characteristics of this drug is an important part of AMS.

- Conversion from Parenteral to Oral Therapy: A systematic plan for parenteral to oral conversion of antimicrobials is important.

- Coordination with Microbiology Laboratory: The clinical microbiology laboratory plays a critical role in AMS by providing patient-specific culture and susceptibility data to optimize individual antimicrobial management and by assisting infection control efforts in the surveillance of resistant organisms and in the molecular epidemiologic investigation of outbreaks.

- Monitoring of Process and Outcome Measurements:Both process measures (did the intervention result in the desired change in antimicrobial use?) and outcome measures (did the process implemented reduce or prevent resistance or other unintended consequences of antimicrobial use?) are useful in determining the impact of AMS on antimicrobial use and resistance patterns.

\section{AMS Policy and Programs in Great Britain (UK)}

The National Health Service (NHS) has declared that "good antimicrobial stewardship is a cornerstone for both effective treatment of infections and reduction of antimicrobial resistance" [84]. The NHS primary objectives for an effective antimicrobial stewardship program include: (i) Optimizing therapy for the individual patient; (ii)Preventing overuse, misuse and abuse; (iii) Minimizing development of resistance in patients and community levels [85].

AMS program guidance is administered through NICE (National Institute for Health and Care Excellence) and provides extensive antibiotic prescribing guidelines for both hospital and outpatient settings. The NICE AMS strategy is labeled as "Start mart - then focus" [85]. The 'Start smart' element states that antibiotics should be started within one hour of diagnosis in patients with severe and life threatening infections, in line with local antibiotic prescribing guidance. In patients with less severe infection, local prescribing guidance recommends narrow spectrum antibiotics that cover the expected pathogens [85]. The focus element states that clinical diagnosis and continuing need for antibiotics should be reviewed within 48 to 72 hours, with 5 options to consider [85]:

1. Stop antibiotics if there is no evidence of infection

2. Switch antibiotic formulation from intravenous to oral

3. Change antibiotic - preferably to a narrower spectrum, but broader if required

4. Continue antibiotics and document next review date

5. Start outpatient parenteral antibiotic therapy 
Example of Successful AMS Program in the UK: An AMS program applied in Northeast Scotland in the United Kingdom was reported in 2015. The program was applied to 1.29 million hospital admissions and to 455,000 primary use-out patient cases. This program resulted in restriction the use of the "4C antibiotics" (cephalosporins, clindamycin, ciprofloxacin and co-amoxiclav) and macrolide antibiotics. The program demonstrated a $47 \%$ decrease in the use of these named antibiotics in hospitals, and a $27 \%$ reduction in antibiotic use in outpatient settings. At the same time, reduced antibiotic use also reduced bacterial infections. The primary outcome reported was prevalence density of MRSA infections per 1,000 occupied bed days (OBD) in hospitals, or per 10,000 inhabitants per day (ID) in the community/out patient setting. The MRSA prevalence density decreased during the AMS program by $54 \%$ in hospitals and by $37 \%$ man reduction in community /out patients [86].

Evaluation of Antimicrobial Stewardship Program in USA: In September 2016 the private pharmacy services company "Comprehensive Services Company (CPS) reported the results of an expansive study conducted for the entire year of 2014 over 180 US hospitals [87].The program reviewed 45 hospitals with antibiotic drug spending greater than $\$ 10$ per patient per day, and also reviewed 135 hospitals with antibiotic drug spending less than $\$ 10$ per patient per day. CPS oversaw an Antimicrobial Stewardship program in these hospitals for one year. The study showed that hospitals that used the CPS designed AMS program had reduced antibiotic usage of $11.4 \%$. CPS offers an AMS service bundle to hospitals, which contains these minimum elements: (i) Hospitals must establish an interdisciplinary approach that includes pharmacy; (ii) Metrics must be reported for recording and evaluation, and (iii) An education and training initiative are included.

\section{Conclusion}

Antimicrobial Resistance (AMR) among gram-negative bacteria has reached critical levels on a global basis. The rise of carbapenemase resistance in Enterobacteriaceae carrying additional resistance genes for multiple antibiotic classes has created a phalanx of organisms that are resistant to all available antimicrobial treatment [88]. Carbapenem Resistant Enterobacteriacea (CRE) infections are known to be associated with significant morbidity and mortality. Some experts have suggested that the global population will face two concomitant and worldwide epidemics of carbapenemase producers [43]. The first epidemic will cover carbapenemase producers as a source of community acquired infections. The second epidemic will cover nosocomial carbapenemase producers in $K$. pneumoniae of all types (KPC, IMP, VIM, NDM and OXA-48).

For the first epidemic, community acquired infections for these carbapenemases are primarily of the NDM and OXA-48 types. It is believed that the number of such cases in disease endemic areas are very high. The spread of CRE producers as in E. coli will parallel the rapid global spread of ESBL producers in the community. In contrast to a viral epidemic (i.e.- pandemic H1N1 in 2009), an epidemic of carbapenemase producers cannot stop spontaneously. This is because there are multiple factors involved that favor propagation, including: lack of hygiene, overuse of antibiotics and increased global travel. In addition, there are many carbapenemase producing organisms that carry unrelated drug-resistance determinants resulting in their spread to both $\beta$-lactam antibiotics and to other antibiotic that are not structurally related to $\beta$-lactams.

The actual prevalence of carbapenemase producers is not clear because many countries that are likely to be their main reservoirs have poor detection and reporting systems in place. As a comparison, the known prevalence rate of ESBL producers in E. coli is estimated to be $3 \%$ to $5 \%$ in France and more than $80 \%$ in India [89]. It is possible that carbapenemase prevalence may show a similar distribution.

For the second epidemic - nosocomial infections, the likely cause will be carbapenemase producers in $K$. Pneumoniae of all types (KPC, IMP, VIM NDM1 and OXA-48. In certain countries, high rates of various types of carbapenemase producers already exist: Greece (VIM \& KPC) and the Indian subcontinent (NDM, KPC, OXA-18) [39,89]. It is believed that K. pneumoniae will be a likely carrier of carbapenemase infections because it has been repeatedly cultured in the most common Enterobacteriacea species for spreading ESBL genes in healthcare facilities during the past 30 years. It is therefore expected that K. Pneumoniae will be the most likely carbapenemase producer found in patients with identical risk factors for ESBL infections: (Patients receiving broad spectrum antibiotic therapy, patients in intensive care units, immunosuppressed patients, and surgical patients). It is therefore an urgent task for early identification of carbapenemase producers (CRE) in clinical infections at the carriage stage in order to prevent the development of hospital outbreaks of infection [43].

The lack of new antibacterial drugs in the development pipeline of pharmaceutical companies is a serious global problem. Carbapenemase producers in Enterobacteriacea are different from other multi-drug resistant bacteria because they are susceptible to few if any antibiotics. Therefore, it is essential that prudent use of antibiotics in clinical and outpatient practice settings be done in order to preserve the therapeutic efficacy of the existing arsenal of antibiotics for as long as possible [11]. Fundamental changes are required in the application of antibiotics for both medical and non- medical applications. A recent study by the British Govt has recommended the following steps to reduce antibiotic demand and consumption:

1. A massive global public awareness campaign to cover both patients and farmers to reduce their demand; and prescribers and clinicians to not prescribe antibiotics when they are not needed.

2. Improve hygiene and prevent the spread of infection. This effort includes improvement in access to clean water and better sanitation in developing countries, and reducing infections in healthcare settings in all countries.

3. Reduce unnecessary use of antibiotics in agriculture and their dissemination into the environment. 
4. Improve global surveillance of drug resistance and antimicrobial consumption in humans and animals. While surveillance is a cornerstone of infectious disease management, it has been under-resourced in the effort to reduce AMR.

5. Promote new rapid diagnostics to reduce unnecessary use of antibiotics.

6. Promote the development and use of vaccines as alternatives. By reducing and preventing infections, demand for therapeutic treatments and also antibiotic use will be reduced. These steps are expected to slow the development and spread of AMR.

7. Increase the number of effective antimicrobial drugs to defeat infections that have become resistant to existing medicines.. The problem is that there is presently insufficient private and public investment in R\&D focused on developing new antimicrobial drugs and fighting AMR [11].

In conclusion, the world faces serious global public health problems from a post antibiotic world Many of the drivers of AMR have a common origin in inappropriate use of antimicrobials in both human and animal health care or in agriculture or from environmental contamination. As a result, AMR continues its global spread. From a strategic view, comprehensive measures are needed to reduce unnecessary antibiotic use in both healthcare and agricultural settings so that these medicines may have an extended service life and continue to be effective against infections for a longer period. From a more immediate tactical view, key steps to prevent the establishment of CRE are early detection through good diagnostic practices and containment of spread through patient and contact screening as well as infection prevention and control measures including antibiotic stewardship [47].

\section{Acknowledgement}

This article is dedicated to Nobel Laureate Sir "Ernst Chain", the founder of the antibiotic era.

\section{References}

1. Friedman T. Antibiotic Resistance Threats in the United States. Centers for Disease Control \& Prevention (CDC), US Department of Health \& Human Services. 2013.

2. Scott II, Douglas R. The Direct Medical Costs of Healthcare Associated Infections in US Hospitals and the Benefits of Prevention. 2009.

3. Kardos N, Demain A. Ernst Chain: a Great Man of Science. Applied Microbiology and Biotechnology. 2013;97(15):6613-6622.

4. World Health Organization (WHO). 2011.

5. Fishman N. Antimicrobial stewardship. Am J Infect Control. 2006;34(5 Suppl 1):S55-63.

6. WHO. The Emerging Threat of Antimicrobial Resistance: Options for Action. 2012.

7. Benedetta Allegranzi, Sepideh Bagheri Nejad, Christophe Combescure, Wilco Graafmans, Homa Attar, Liam Donaldson, et al. Burden of endemic health-care-associated infections in developing countries: systemic review and meta-analysis. The Lancet. 2011;377(9761):228241.
8. Kardos N. Overuse of Antibiotics: Non-Medical Applications. Antibiotics: Current Innovations \& Future Trends. Chapter 8. UK : Caister Academic Press; 2015. p. 113-146.

9. Swinford S. Too many antibiotics are creating health disaster, doctors told Telegraph UK. 2015.

10.UK 5 Year Antibiotic Resistance Strategy 2013 to 2018. Dept of Health. HM Government, London; 2013.

11. O'Neill J. Tackling Drug-Resistant Infections Globally: Final Report and Recommendations. The Review on Antimicrobial Resistance. Dept of Health,HM Government, London; 2016.

12. Antibiotic Resistance: Ecological Perspective on an Old Problem. American Society for Microbiology. 2009.

13. Laxminarayan R, Duse A, Wattal C, Zaidi AK, Wertheim HF, Sumpradit N, et al. Antibiotic resistance-the Need for Global Solutions. Lancet Infect Dis. 2013;13(12):1057-1098. doi: 10.1016/S1473-3099(13)70318-9

14. Bud R. Penicillin, Triumph \& Tragedy. Oxford University Press. UK; 2007.

15. North EA, Christie R. Observations on the Sensitivity of Staphylococci to Penicillin. Medical Journal of Australia. 1945;2(2):44-46.

16. Bugnon-Reber A, de Torrenté A, Troillet N, Genné D. Antibiotic Misuse in Medium-Size Swiss Hospitals. Swiss Med Wkly. 2004;134(3334):481-485.

17. Steinman MA, Landefeld CS, Gonzales R. et al. Prediction of Broad Spectrum Antibiotic Prescribing for Acute respiratory Tract Infections in Adult Primary Care. JAMA. 2003;289(6):719-725.

18. ECDC/EMEA Joint Technical Report: The bacterial challenge: time to react. 2009.

19. Livermore DM. "Multiple mechanisms of Antimicrobial Resistance in Pseudomonas aeruginosa: Our Worst Nightmare? Clin Infect Dis. 2002;34(5):634-640

20. Pendleton JN, Gorman SP, Gilmore BF. Clinical relevance of the ESKAPE pathogens. Expert Rev Anti Infect Ther. 2013;11(3):297-308. doi: 10.1586/eri.13.12

21. Woodford N, Wareham DW, Guerra B, Teale C. Carbapenemase producing Enterobacteriaceae and non-Enterobacteriaceae from animals and the environment: an emerging public health risk of our own making? J Antimicrob Chemother. 2014;69(2):287-291. doi: $10.1093 / \mathrm{jac} / \mathrm{dkt} 392$

22.Thomas KS. Extended-Spectrum- $\beta$-lactamase, AmpC, and Carbapenemase Issues. J Clin Microbiol. 2010;48(4):1019-1025. doi: 10.1128/JCM.00219-10

23. Boucher HW, Talbot GH, Bradley JS, Edwards JE, Gilbert D, Rice LB, et al. Bad Bugs, no Drugs: No ESKAPE! An Update from the Infectious Diseases Society of America. Clin Infect Dis. 2009;48(1):1-12. doi: $10.1086 / 595011$

24. Centers for Disease Control and Prevention (CDC). FAQ about choosing and Implementing a CRE definitions. 2015.

25. Magiorakos AP, Srinivasan A, Carey RB, Carmeli Y, Falagas ME, Giske $\mathrm{CG}$, et al. Multidrug-resistant extensively drug-resistant and pandrugresistant bacteria: an international expert proposal for interim standard definitions for acquired resistance. Clin Microbiol Infect. 2012;18(3):268-281. doi: 10.1111/j.1469-0691.2011.03570.x

26. Queenan AM, Bush K. Carbapenemases: the versatile $\beta$-lactamases. Clin Microbiol Rev. 2007;20(3):440-458.

Citation: Kardos N (2017) Overuse of Antibiotics and Antibiotic Resistance in Medical Applications Featuring CarbapenemasePage 19 of 21 Resistant Enterobacteriaceae (CRE). SOJ Microbiol Infect Dis 5(5):1-21. 
27. Bush K, Jacoby GA. Updated Functional Classification of $\beta$-Lactamases. Antimicrob Agents Chemother. 2010;54(3):969-976. doi: 10.1128/ AAC.01009-09

28. Kani SS, Kanafani ZA. Current Concepts in Antimicrtobial Therapy Against Resistant Gram-Negative Orgamnisms: Exteended-Spectrum $\beta$-Lactamase-Producing Enterobacteriaceae, Carbapenem-Resistant Enterobacteriaceae, and Multidrug-Resistant Enterobacteriaceae, and Multidrug-resistant Pseudomonas aeruginosa. Mayo Clin Proc. 2011;86(3):250-259. doi: 10.4065/mcp.2010.0674

29. Tängden MA, Giske CG. Global dissemination of extensively drugresistant carbapenemase-producing Enterobacteriaceae: clinical perspectives on detection, treatment and infection control. J Intern Med. 2015;277(5):501-512. doi: 10.1111/joim.12342

30. Kanj SS, Kanafani ZA. Current concepts in antimicrobial therapy against resistant gram-negative organisms: extended-spectrum beta-lactamase-producing Enterobacteriaceae, carbapenemresistant Enterobacteriaceae, and multidrug-resistant Pseudomonas aeruginosa. Mayo Clin Proc. 2011;86(3):250-259. doi: 10.4065/ mcp.2010.0674

31. Doi YH, Paterson DL. Carbapenemase-Producing Enterobacteriaceae. Semin Respir Crit Care Med. 2015;36(1):74-84. doi: 10.1055/s-00351544208

32. Lee CR, Lee JH, Park KS, Kim YB, Jeong BC, Lee SH. Global Dissemination of Carbapenemase-Producing Klebsiella pneumoniae: Epidemiology, Genetic Context, Treatment Options, and Detection Methods. Front Microbiol. 2016;7:895. doi: 10.3389/fmicb.2016.00895

33. Shah B, Foodad F. The ABCs on ESBLs and CREs: A Pharmacist's Primer on Multi-Drug Resistant Gram-Negative Organisms. Presentation at Penna. Pharm Assoc. 2014.

34. Bassetti M, Ginocchio F, Mikulska M, Taramasso L, Giacobbe DR. Will new antimicrobials overcome resistance among gram-negatives? Expert Rev Anti Infect Ther. 2011;9(10):909-922. doi: 10.1586/ eri.11.107

35. Rishi H, Dhillon P, Clark J. ESBLs: A Clear and Present Danger? Critical Care Research and Practice. 2012;11.

36. Papp-Wallace KM, Endimiani A, Taracila MA, Bonomo RA. Carbapenems: Past, Present and Future. Antimicrob Agents Chemother. 2011;55(11):4943-4960. doi: 10.1128/AAC.00296-11

37.Zavascki AP, Bullita JB, Landersdorfer CB. Combination Therapy for Carbapenem-resistant Gram-Negative Bacteria. Expert Rev Anti Infect Ther. 2013;11(12):1333-1353. doi: 10.1586/14787210.2013.845523

38. Martinez-Lacasa J, Garaau J. The role of carbapenems in the treatment of nosocomial infection. Enferm Infecc Microbiol Clin. 1997;15 Suppl 1:78-85.

39. Patel N, Harrington S, Dihmess A, Woo B, Masoud R, Martis P, et al. Clinical epidemiology of carbapenem-intermediate or -resistant Enterobacteriaceae. J Antimicrob Chemother. 2011;66(7):1600-1608. doi: $10.1093 / j a c / d k r 156$

40. European Centre for Disease Prevention and Control. Risk assessment on the spread of Carbapenemase-producing Enterobacteriaceae (CPE) through patient transfer between healthcare facilities, with special emphasis on cross-border transfer. 2011.

41. Hussein K, Sprecher H, Mashiach T, Oren I, Kassis I. Finkelstein R.
Carbapenem resistance among Klebsiella pneumoniae-infections: a matched case control study.J Antimicrob Chemother. 2007;60(5):11241130.

42. McLaughlin M, Advincula MR, Malczynski M, Qi C, Bolon M, Scheetz MH. Correlation of antibiotic use and carbapenem resistance in Enterobacteriaceae. Antimicrob Agents Chemother. 2013;57(10):5131-5133. doi: 10.1128/AAC.00607-13

43. Samuelson Ø, Naseer U, Tofeland S, Skutlaberg DH, Onken A, Hjetland $\mathrm{R}$, et al. Emergence of clonally related Klebsiella pneumoniae isolates of sequence type 258 producing plasmid-mediated KPC carbapenemase in Norway and Sweden. J Antimicrob Chemother. 2009;63(4):654-658. doi: $10.1093 / \mathrm{jac} / \mathrm{dkp} 018$

44. Nordman P, Naas T, Poires L. Global spread of carbapenemaseproducing enterobacteriaceae. Emerging Infectious Diseases. 2011.

45. Sidjabat H, Nomma GR, Walsh TR, Binotta E, Htion A, Hayashi Y, et al. Carbapenem resistance in Klebsiella pneumoniae due to the New Delhi Metallo- $\beta$-Lactamase. Clin Infect Dis. 2011;52(4):481-484. doi: $10.1093 / \mathrm{cid} / \mathrm{ciq} 178$

46. Gandra S, Mojica N, Klein EY, Ashok A, Nerurkar V, Kumari M, et al. Trends in antibiotic resistance among major bacterial pathogens isolated from blood cultures tested at a large private laboratory network in India, 2008-2014. Int J Infect Dis. 2016;50:75-82. doi: 10.1016/j.jid.2016.08.002

47. Laxminarayan R, Matsoso P, Pant S, Brower C, Røttingen JA, Klugman K, et al. Access to effective antimicrobials: a worldwide challenge. Lancet. 2016;387(10014):168-175. doi: 10.1016/S0140-6736(15)00474-2

48. European Centre for Disease Prevention and Control. Carbapenemaseproducing bacteria in Europe: interim results from the European Survey on carbapenemase-producing Enterobacteriaceae (EUSCAPE) project. 2013.

49. Marsik FJ, Nambiar S. Review of Carbapenemases and AmpC-beta Lactamases. Pediatr Infect Dis J. 2011;30(12):1094-1095. doi: 10.1097/INF.0b013e31823c0e47

50. Center for Disease Dynamics, Economics \& Policy. The State of the World's Antibiotics. 2015.

51. Baggs J, Fridkin SK, Pollack LA, Srinivasan A, Jernigan JA. Estimating National Trends in Inpatient Antibiotic Use Among US Hospitals From 2006 to 2012. JAMA Intern Med. 2016;176(11):1639-1648. doi: 10.1001/jamainternmed.2016.5651

52. Ganguly NK. Situation Analysis: Antibiotic Use and Resistance in India. Center for Disease Dynamics, Economics \& Policy. 2011.

53. Harris PN, Tambyah PA, Paterson DL. $\beta$-lactam and $\beta$-lactamase inhibitor combinations in the treatment of extended-spectrum $\beta$-lactamase producing Enterobacteriaceae: time for a reappraisal in the era of few antibiotic options? Lancet Infect Dis. 2015;15(4):475485. doi: 10.1016/S1473-3099(14)70950-8

54. Carlet J, Jarlkier V, Narbarth S, Voss A, Groosens H, Pittet D. Ready for a world without antibiotics? The Pensières Antibiotic Resistance Call to Action. Antimicrob Resist Infect Control. 2012;1(1):11.

55. Tarlington K. Antibiotic Use in Outpatient Setting. The Pew Charitable Trust, Philadelphia. 2015.

56. The White House. National Action Plan for Combating AntibioticResistant Bacteria. 2015.

Citation: Kardos N (2017) Overuse of Antibiotics and Antibiotic Resistance in Medical Applications Featuring CarbapenemasePage 20 of 21 Resistant Enterobacteriaceae (CRE). SOJ Microbiol Infect Dis 5(5):1-21. 
57. Obama. Executive Order: Combating Antibiotic-Resistant Bacteria. The White House. 2014.

58. CDC. Antibiotic Resistance Solutions Initiative. 2015.

59. Chin JL, Kong DCM, Stuart RL. Reducing inappropriate antibiotic prescribing in the residential care setting: current prospectives. Clin Interv Aging. 2014;9:165-177. doi: 10.2147/CIA.S46058

60. Center for Disease Prevention and Control. Antibiotic use in nursing homes. 2013.

61. Doyle K. Overuse of Nursing Home Antibiotics May Put All Residents at Risk. Medscape. 2015.

62. Hughes CM, Tunney MM. Improving prescribing of antibiotics in longterm care. JAMA Intern Med. 2013;173(8):682-683.

63. McKay B. New Push to Stop Overuse of Antibiotics in Nursing Homes. The Wall Street Journal. 2015.

64. Stone S. Core Elements of Antibiotic Stewardship for Nursing Homes. Center for Disease Control and Prevention (CDC). Medscape. 2015.

65. Van Boeckel TP, Gandra S, Ashok A, Quentin Caudron, Bryan T Grenfell, Simon A Levin, et al. Global antibiotic consumption 2000 to 2010: an analysis of national pharmaceutical sales data. The Lancet Infectious Diseases. 2014;14(8):742-750.

66. Kumarasamy KK, Toleman MA, Walsh TR, Bagaria J, Butt F, Balakrishnan $\mathrm{R}$, et al. Emergence of a New Antibiotic resistance Mechanism in India, Pakistan and the UK.: A Molecular Biology and Epidemiological Study. Lancet Infect Dis. 2010;10(9):597-602. doi: 10.1016/S14733099(10)70143-2

67.Spatuzza A. Antibiotic Resistance: Are we Killing the Cure? Perspectives in Health Magazine, the Magazine of the Pan American Health Organization. 2002; 7(1).

68. Ganguly NK, Arora NK, Chandy SJ, Fairoze MN, Gill JP, Gupta U, et al. Rationalizing antibiotic use to limit antibiotic resistance in India Indian J Med Res. 2011;134:281-294.

69. Morgan DJ, Okeke IN, Laxminarayan R, Perencevich EN, Weisenberg S. Non-prescription antimicrobial use worldwide: a systematic review. Lancet Infect Dis. 2011;11(9):692-701. doi: 10.1016/S14733099(11)70054-8

70. Fair R, Tor Y. Antibikotics and Bacterial Resistance in the 21st Century. Perspect Medicin Chem. 2014;6:25-64. doi: 10.4137/PMC.S14459

71. Oberol L, Singh N, Sharma P, Aggarwal A. ESBL, MBL and Ampc $\beta$ Lactamases Producing Superbugs - Havoc in the Intensive Care Units of Punjab India. J Clin Diagn Res. 2013;7(1):70-73. doi: 10.7860/ JCDR/2012/5016.2673

72. IMS. Advancing the responsible use of medicines. Applying levers for change. Right medicine for the right patient- optimize antibiotic use. Institute for Healthcare Informatics.
73.Sun Q, Yin J, Zuo GY, Meng QY. The irrational use of drugs in rural China: evidence from two provinces. Third International Conference for Improving Use of Medicines. 2011.

74.van der Bij AK., Pitout JD. The role of international travel in the worldwide spread of multiresistant Enterobacteriacea. J Antimicrob Chemother. 2012;67(9):2090-2100. doi: 10.1093/jac/dks214

75. Karami G, Chastre J, Wilcox MH, Vincent JL. Antibiotic strategies in the era of multidrug resistance. Crit Care. 2016;20(1):136. doi: 10.1186/ s13054-016-1320-7

76. Sharland M. WHO Essential Medicines List Update: Thoughts from an Expert. 2017.

77. WHO Model List of Essential Medicines. 2017.

78. Aiken M, Valkova S, Avoidable Costs in U.S. Healthcare. The $\$ 200$ Billion Opportunity from Using Medications More responsible. IMS Institute for Healthcare Infomatics, Parsippany New Jersey. 2013.

79. Core Elements of Hospital Antibiotic Stewardship Programs. Centers for Disease Control and Prevention.

80. Tamma PD, Holmes A, Ashley ED. Antimicrobial stewardship: another focus for patient safety? Curr Opin Infect Dis. 2014;27(4):348-355. doi: 10.1097/QCO.0000000000000077

81. Murray A, Antimicrobial Stewardship, Pharmacist's Letter / Prescriber's Lette. 2015.

82. Dellit TH, Owens RC, McGowan JE Jr, Gerding DN, Weinstein RA, Burke JP, et al. Infectious Disease Society of America and the Society for Healthcare Epidemiology of America: guidelines for developing an institutional program to enhance antimicrobial stewardship. Clin Infect Dis. 2007;44(2):159-177.

83. Rahal JJ, Urban C, Horn D, Freeman K, Segal-Maurer S, Maurer J, et al. Class restriction of cephalosporin use to control cephalosporin resistance in nosocomial Klebsiella. JAMA. 1998;280(14):1233-1237.

84. Core elements of hospital antibiotic stewardship programs. CDC. 2014.

85. Antimicrobial stewardship: prescribing antibiotics. NICE (National Institute for Heath and Care Excellence). 2015.

86. Dixon J, Duncan CJ. Importance of Antimicrobial Stewardship to the English National Health Service. Infect Drug Resist. 2014;7:145-152. doi: $10.2147 /$ IDR.S39185

87. Two year antibiotic study in 180 U.S. hospitals reveals $11 \%$ reduction in antibiotic use through antimicrobial stewardship program. Comprehensive Pharmacy Services, Memphis TN. 2016.

88. Logan LK. Carbapenem-Resistant Enterobacteriaceae: An Emerging Problem in children. Clin Infect Dis. 2012;55(6):852-859. doi: $10.1093 / \mathrm{cid} / \mathrm{cis} 543$

89. Jean SS, Hsuech RR. High burden of antimicrobial resistance in Asia. Int J Antimicrob Agents. 2011;37(4):291-295. doi: 10.1016/j. ijantimicag.2011.01.009 